\title{
Health Implications of Judo Training
}

\author{
Patrik Drid $^{1, *(\mathbb{D}}$, Emerson Franchini ${ }^{2} \mathbb{D}$, João Paulo Lopes-Silva ${ }^{3} \mathbb{D}$, David H. Fukuda $4 \mathbb{C}^{\mathbb{D}}$, Adam J. Wells $4 \mathbb{D}^{\mathbb{D}}$,

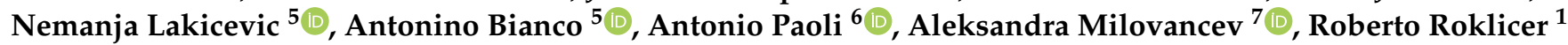 \\ and Tatjana Trivic ${ }^{1}$
}

1 Faculty of Sport and Physical Education, University of Novi Sad, 21000 Novi Sad, Serbia; roklicer.r@gmail.com (R.R.); ttrivic@yahoo.com (T.T.)

2 School of Physical Education and Sport, University of São Paulo, São Paulo 05508-030, Brazil; efranchini@usp.br

3 Applied Research Group to Performance and Health, CESMAC University Center, Maceió 57051-160, Brazil; joao.lopes@cesmac.edu.br

4 School of Kinesiology and Physical Therapy, Institute of Exercise Physiology and Rehabilitation Science, University of Central Florida, Orlando, FL 32816, USA; david.fukuda@ucf.edu (D.H.F.); adam.wells@ucf.edu (A.J.W.)

5 Sport and Exercise Sciences Research Unit, University of Palermo, 90144 Palermo, Italy; lakinem89@gmail.com (N.L.); antonino.bianco@community.unipa.it (A.B.)

6 Department of Biomedical Sciences, University of Padova, 35100 Padova, Italy; antonio.paoli@unipd.it

7 Faculty of Medicine, University of Novi Sad, 21000 Novi Sad, Serbia; aleksandra.milovancev@mf.uns.ac.rs

* Correspondence: patrikdrid@gmail.com

check for updates

Citation: Drid, P.; Franchini, E.; Lopes-Silva, J.P.; Fukuda, D.H.; Wells, A.J.; Lakicevic, N.; Bianco, A.; Paoli, A.; Milovancev, A.; Roklicer, R.; et al. Health Implications of Judo Training. Sustainability 2021, 13, 11403. https:// doi.org/10.3390/su132011403

Academic Editors: Pablo Jorge

Marcos-Pardo, Noelia

González-Gálvez and Lotfi Aleya

Received: 23 July 2021

Accepted: 11 October 2021

Published: 15 October 2021

Publisher's Note: MDPI stays neutral with regard to jurisdictional claims in published maps and institutional affiliations.

Copyright: (c) 2021 by the authors. Licensee MDPI, Basel, Switzerland. This article is an open access article distributed under the terms and conditions of the Creative Commons Attribution (CC BY) license (https:// creativecommons.org/licenses/by/ $4.0 /)$.

\begin{abstract}
Although current physical activity (PA) guidelines regarding aerobic and anaerobic training are helpful for the population at large, many individuals prefer to engage in alternative forms of PA such as combat sports. As both a martial art and sport, judo is a physically demanding form of PA that potentially offers a novel experience, consequently leading to greater PA adherence. This study aimed to thoroughly search the existing literature to determine the health benefits of judo-specific training. PubMed and Web of Science were searched for original research studies. Only peer-reviewed studies that examined the effects of judo training in males and females aged 18-35 were included in the study. Out of 507 potentially relevant studies, 84 studies met our inclusion criteria and were further analyzed. Data showed that judo athletes and recreational judo practitioners show above-average $\mathrm{VO}_{2 \max }$, improved body composition, increased bone mineral density, and bone mineral content. Judo is associated with a sequence of adaptations in cardiac structure, function, and blood pressure changes. More research is needed to discover if these changes are maladaptive.
\end{abstract}

Keywords: aerobic power; respiratory function; obesity; bone mineral density; arterial hypertension; cardiac remodeling

\section{Introduction}

Being physically active is widely accepted as an effective way to promote health and prevent an incredible spectrum of health risk factors within all age, gender, ethnic, and socioeconomic subgroups [1]. According to the World Health Organization, "health is a state of complete physical, mental and social well-being and not merely the absence of disease or infirmity [2]." Based on the ever-growing evidence of beneficial impacts of regular physical activity (PA) on overall health, it is undeniable that PA is one of the key pillars of good health and longevity in humans. Despite the well-documented benefits associated with sustained PA [1], recent evidence shows that levels of PA in different populations remain relatively low, creating an environment that further exacerbates the obesity epidemic present on a global scale [3]. Thus, it is critical to identify approaches to alleviate the inactivity crisis, which will bring about an increase in intentional PA and a decrease in sedentary pursuits in the overall population. According to the latest PA recommendations put together by the American College of Sports Medicine and American 
Heart Association, adults should engage in at least $150 \mathrm{~min}$ of aerobic activity per week [4]. In addition, adults are advised to participate in resistance training (using major muscle groups) two times per week while trying to reach volitional fatigue.

Although these recommendations are helpful for the population at large, many individuals aspire to go beyond typical PA, and partake in alternative forms of PA. Some evidence suggests that novelty can contribute to greater long-term adherence among individuals participating in PA [5], one of the most important therapeutic agents combating obesity. In recent years, there has been a steady increase in people actively participating in combat sports, with judo being one of the most popular forms [6]. Since being included as a demonstration sport in 1964 and a full Olympic sport in 1972, judo has gained considerable attention worldwide [7]. Judo is a very demanding type of PA that requires an involvement of the entire body and a modifiable set of technical-tactical skills $[8,9]$. As such, judo has the potential to be a type of exercise that can positively influence one's health, regardless of age and gender. These positive responses are dictated in part by a number of training variables, such as intensity, frequency, and volume, inherent to the practice of judo. Even though data on the health benefits of exercise, in general, is abundant, little is known about whether there is a direct relationship between improved health and participation in judo regularly. Therefore, the goal of this study was to review the existing literature to investigate the potential benefits of judo participation on several risk factors and significant diseases related to metabolic health, including low fitness levels, obesity, hypertension, hyperlipidemia, and cardiovascular disease.

\section{Materials and Methods}

\subsection{Search Strategy}

To ensure transparent and accurate reporting, we followed the preferred reporting items for systematic reviews and meta-analyses (PRISMA) statement and, for this manuscript, the PRISMA Protocol [10]. PubMed and Web of Science were searched via EndNote 9.1 software (Clarivate Analytics, Jersey, UK) by two experienced researchers. The following search terms were used via the Boolean operator method to generate potential studies: "physical fitness"; "aerobic fitness"; "fitness profile"; "cardiovascular disease"; "heart disease"; "cardiac function"; "hypertension"; "blood pressure"; "pulmonary function"; "respiratory function"; "lipid profile"; "obesity"; “body composition"; "bone health"; "hormone concentrations"; and "inflammatory mediators". Each of these terms was used in combination with "judo". The initially identified studies were manually screened against eligibility according to a three-step process: title; abstract; and full-text selection. The two researchers worked independently, although they were not blinded to the studies. In case of disagreements between the two researchers, a third investigator (full professor) intervened and decided. The screening process is summarized in a PRISMA flow diagram displayed in Figure 1.

\subsection{Inclusion and Exclusion Criteria}

Only original articles written in English and published in peer-reviewed journals from 1996 to July 2021 were considered for this review. The examined population were healthy males and females aged 18-35 partaking only in judo-specific training either professionally or recreationally. Included studies had to report on data in accordance to health domains delineated in the aforementioned search terms. Moreover, studies had to be cohort studies (longitudinal or cross-sectional), case-control studies, or experimental studies. Various formats of publications, such as reviews, meta-analyses, abstracts, citations, scientific conference abstracts, opinion pieces, books, book reviews, statements, letters, editorials, non-peer reviewed journal articles, and commentaries were excluded. 


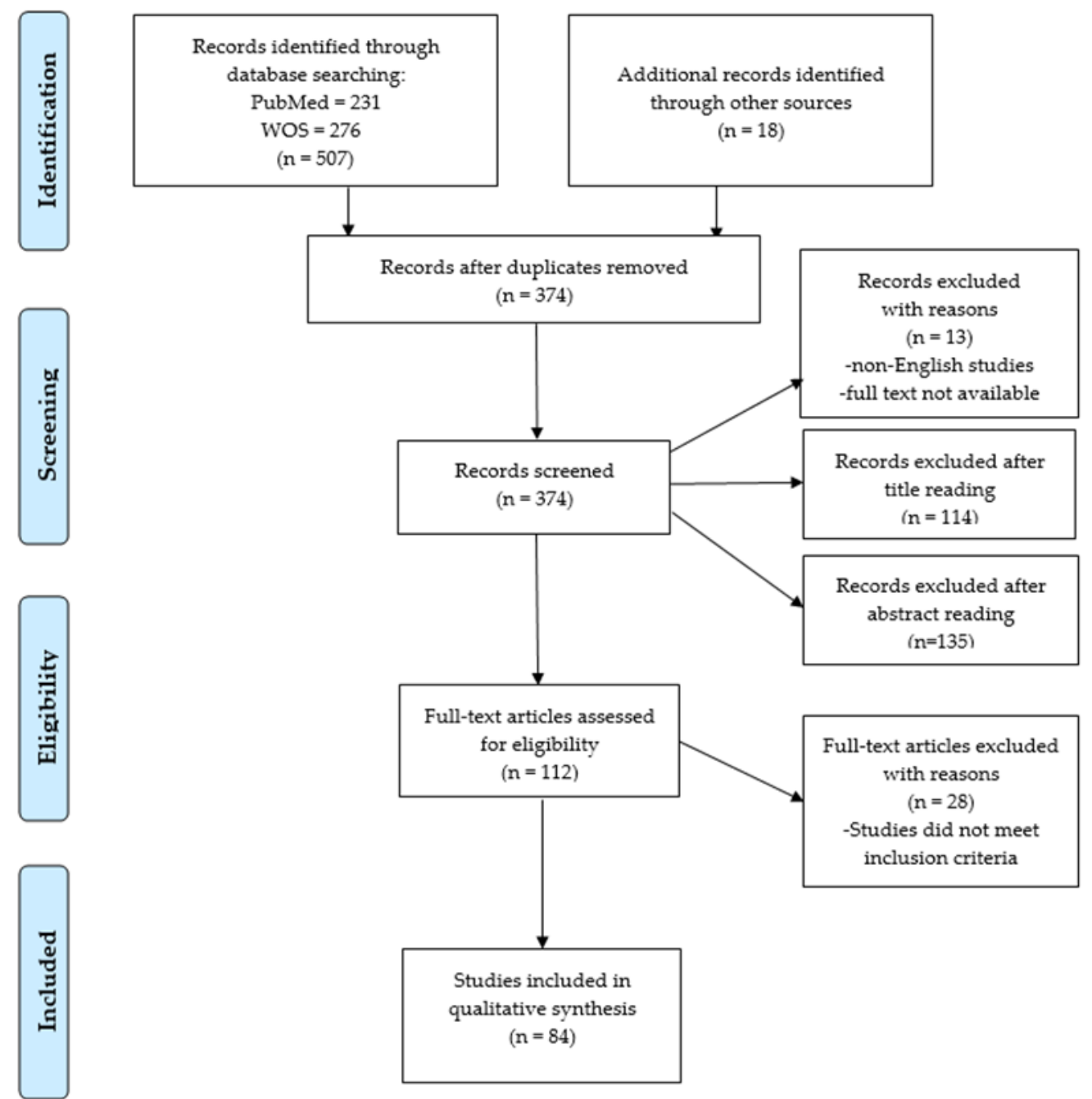

Figure 1. PRISMA Flow Diagram.

\subsection{Data Extraction}

Essential characteristics of all included studies (height, weight, combat sport, variable of interest, etc.) were depicted in tables using a Microsoft Excel 2013 (Microsoft Corp, Redmond, Washington, DC, USA) spreadsheet. Acquired data were grouped in line with the body systems assessed within judo training-related studies. In addition, certain characteristics that went beyond tabular explanation were described narratively.

\subsection{Risk of Bias Assessment}

The Newcastle-Ottawa Scale for assessment of quality of nonrandomized studies [11] was used in the present review. This scale has been validated previously and explores quality in three broad perspectives: study groups selection; the comparability of the groups; and the ascertainment of either the exposure or outcome of interest for case-control or cohort studies, respectively. Two independent researchers completed the scale for the included studies to determine the quality of each study. The interclass correlation statistical method was used to determine inter-rater reliability via SPSS (IBM, New York, NY, USA, v.20).

\section{Results}

The preliminary PubMed and Web of Science database search generated 507 potentially relevant studies. Additionally, 18 articles that were not found through our initial search, but were thought to be relevant, were added for the full-text analysis. These articles were in 
alignment with predefined inclusion criteria, and were identified through bibliographies of already included studies. Then, the software eliminated 151 duplicates, leaving 374 articles to be further analyzed. Thirteen articles were removed due to only the non-English versions being available and a lack of full-text availability. All remaining articles were screened in a three-step process where 114 articles were excluded after title reading, 135 were excluded after abstract reading, and 27 articles were excluded after full-text reading. A total of 84 articles met our inclusion criteria and were analyzed in-depth for further data synthesis. The biggest study design flaw noted within virtually all of the included studies was the lack of the control group. We have also noted huge disparity in the sample size, length of the study, training load per week, etc. Many studies did not report on the sporting level of the athletes involved, or their previous experience in judo. Some of the subjects included were not truly representative when it comes to the exposed cohort, meaning that they were practicing judo on a recreational basis, and were not professional athletes. Overall, the studies were of fair quality (standards posed by the authors of the Newcastle-Ottawa Scale). The inter-rater reliability between the two researchers was a score of 0.8 , which is considered relatively well).

\subsection{Aerobic Power}

Table 1 presents the maximal oxygen consumption of judo athletes and practitioners. From the included studies, 14 conducted direct $\mathrm{VO}_{2 \max }$ measurements using lower-body and upper-body cycle ergometer tests [12-25], 14 using maximal graded exercise treadmill tests [26-39], and 1 using a judo-specific test [25], whereas 10 estimated the $\mathrm{VO}_{2 \max }$ of the judo athletes and practitioners, primarily using the multistage 20-m shuttle run test [40-49].

Table 1. Maximal oxygen consumption of judokas and judo practitioners.

\begin{tabular}{|c|c|c|c|}
\hline Study & Group Characteristics & Test and Measurement Characteristics & $\underset{\text { Specified Otherwise })}{\mathrm{VO}_{2 \max }\left(\mathrm{mL} \cdot \mathrm{kg}^{-1} \cdot \mathrm{min}^{-1} \text { Unless }\right.}$ \\
\hline $\begin{array}{l}\text { Ahmaidi et al. } \\
\text { [12] }\end{array}$ & Healthy practitioners & $\begin{array}{l}\mathrm{VO}_{2 \max } \text { was measured directly during a } \\
\text { maximal graded exercise cycle } \\
\text { ergometer test. }\end{array}$ & $52.0 \pm 5.3$ \\
\hline \multirow{2}{*}{ Suay et al. [13] } & \multirow{2}{*}{ Adult judokas (winners, $n=14$; losers, $n=14$ ) } & \multirow{2}{*}{$\begin{array}{l}\mathrm{VO}_{2 \max } \text { was measured directly during a } \\
\text { maximal graded exercise cycle } \\
\text { ergometer test. }\end{array}$} & Winners $=52.8 \pm 0.8$ \\
\hline & & & Losers $=50.4 \pm 1.11$ \\
\hline \multirow{20}{*}{$\begin{array}{c}\text { Borkowski et al. } \\
\text { [14] }\end{array}$} & \multirow{20}{*}{$\begin{array}{l}\text { Polish judo team (1994-1997 males, } n=58 \\
\text { 1994-1997, females, } n=48 ; 1998-1999, \text { males, } \\
n=17 ; 1998-1999 \text { females, } n=18 ; 1994-1999 \\
\text { males, } n=75 ; 1994-1999, \text { females, } n=67)\end{array}$} & \multirow{20}{*}{$\begin{array}{l}\mathrm{VO}_{2 \max } \text { was measured directly during a } \\
\text { maximal graded exercise cycle } \\
\text { ergometer test. }\end{array}$} & Males \\
\hline & & & 1994-1997 \\
\hline & & & $<60 \mathrm{~kg}$ up to $>95 \mathrm{~kg}=56.6 \pm 5.6$ \\
\hline & & & $<60 \mathrm{~kg}$ up to $<95 \mathrm{~kg}=57.6 \pm 4.6$ \\
\hline & & & $>95 \mathrm{~kg}=45.2 \pm 3.9$ \\
\hline & & & 1998-1999 \\
\hline & & & $<60 \mathrm{~kg}$ up to $<100 \mathrm{~kg}=55.6 \pm 3.2$ \\
\hline & & & 1994-1999 \\
\hline & & & Principal $=54.5 \pm 4.9$ \\
\hline & & & Reserves $=54.4 \pm 5.6$ \\
\hline & & & Females \\
\hline & & & 1994-1997 \\
\hline & & & $<48 \mathrm{~kg}$ up to $>72 \mathrm{~kg}=49.9 \pm 6.6$ \\
\hline & & & $<48 \mathrm{~kg}$ up to $<72 \mathrm{~kg}=50.7 \pm 5.5$ \\
\hline & & & $>72 \mathrm{~kg}=39.5 \pm 12.0$ \\
\hline & & & 1998-1999 \\
\hline & & & $<48 \mathrm{~kg}$ up to $<78 \mathrm{~kg}=49.9 \pm 4.8$ \\
\hline & & & 1994-1999 \\
\hline & & & Principal $=48.6 \pm 8.6$ \\
\hline & & & Reserves $=47.2 \pm 6.0$ \\
\hline
\end{tabular}


Table 1. Cont.

\begin{tabular}{|c|c|c|c|}
\hline Study & Group Characteristics & Test and Measurement Characteristics & $\begin{array}{c}\mathrm{VO}_{2 \max }\left(\mathrm{mL} \cdot \mathrm{kg}^{-1} \cdot \mathrm{min}^{-1} \text { Unless }\right. \\
\text { Specified Otherwise })\end{array}$ \\
\hline $\begin{array}{l}\text { Degoutte et al. } \\
{[15]}\end{array}$ & $\begin{array}{l}\text { French interregional level male adult judokas } \\
\qquad(n=16)\end{array}$ & $\begin{array}{l}\mathrm{VO}_{2 \max } \text { was measured directly during a } \\
\text { maximal graded exercise cycle } \\
\text { ergometer test. }\end{array}$ & $55.0 \pm 0.5$ \\
\hline $\begin{array}{l}\text { Salvador et al. } \\
{[16]}\end{array}$ & Adult judokas $(n=17)$ & $\begin{array}{l}\mathrm{VO}_{2 \max } \text { was measured directly during a } \\
\text { maximal graded exercise cycle } \\
\text { ergometer test. }\end{array}$ & $3.38 \pm 0.11 \mathrm{~L} \cdot \mathrm{min}^{-1}$ \\
\hline $\begin{array}{l}\text { Degoutte et al. } \\
{[17]}\end{array}$ & $\begin{array}{l}\text { French interregional level male adult judokas } \\
\qquad(n=16)\end{array}$ & $\begin{array}{l}\mathrm{VO}_{2 \max } \text { was measured directly during a } \\
\text { maximal graded exercise cycle } \\
\text { ergometer test. }\end{array}$ & $55.0 \pm 2.9$ \\
\hline $\begin{array}{l}\text { Cottin et al. } \\
{[18]}\end{array}$ & $\begin{array}{l}\text { French national-level male adult judokas } \\
\qquad(n=10)\end{array}$ & $\begin{array}{l}\mathrm{VO}_{2 \max } \text { was measured directly during a } \\
\text { maximal graded exercise cycle } \\
\text { ergometer test. }\end{array}$ & $44.5 \pm 6.0$ \\
\hline \multirow{2}{*}{$\begin{array}{l}\text { Laskowski et al. } \\
\text { [19] }\end{array}$} & \multirow{2}{*}{$\begin{array}{l}\text { Adult judokas with more than ten years of } \\
\text { practice (males, } n=20 \text {; females, } n=15 \text { ) }\end{array}$} & \multirow{2}{*}{$\begin{array}{c}\mathrm{VO}_{2 \max } \text { was measured directly during } \\
\text { the maximal graded exercise cycle } \\
\text { ergometer test. }\end{array}$} & Males $=57.6 \pm 2.3$ \\
\hline & & & Females $=51.2 \pm 4.3$ \\
\hline \multirow{2}{*}{ Mala et al. [20] } & \multirow{2}{*}{$\begin{array}{l}\text { Czech national team (junior, } n=10 \text {; senior, } \\
\qquad n=9 \text { ) }\end{array}$} & \multirow{2}{*}{$\begin{array}{l}\mathrm{VO}_{2 \max } \text { was measured directly during } \\
\text { maximal graded exercise cycle } \\
\text { ergometer test. }\end{array}$} & Junior $=53.58 \pm 4.74$ \\
\hline & & & Senior $=55.00 \pm 3.54$ \\
\hline \multirow{2}{*}{$\begin{array}{l}\text { Kujach et al. } \\
\text { [21] }\end{array}$} & \multirow{2}{*}{$\begin{array}{l}\text { Highly trained judokas ( } n=12, n=6 \text { males } \\
\text { and } n=6 \text { females) }\end{array}$} & \multirow{2}{*}{$\begin{array}{l}\mathrm{VO}_{2 \max } \text { was measured directly during } \\
\text { maximal graded exercise cycle } \\
\text { ergometer test. }\end{array}$} & Males $=52 \pm 4.7^{*}$ \\
\hline & & & Females $=42.5 \pm 5.8$ \\
\hline \multirow{2}{*}{ Julio et al. [22] } & \multirow{2}{*}{ Adult male judokas $(n=12)$} & \multirow{2}{*}{$\begin{array}{l}\mathrm{VO}_{2 \max } \text { was measured directly during a } \\
\text { maximal graded exercise upper-body } \\
\text { and lower-body cycle ergometer test. }\end{array}$} & Upper-body $=37.94 \pm 3.54$ \\
\hline & & & Lower-body $=50.66 \pm 7.17$ \\
\hline \multirow{2}{*}{$\begin{array}{l}\text { Lopes-Silva } \\
\text { et al. [23] }\end{array}$} & \multirow{2}{*}{$\begin{array}{l}\text { Adult regional and national level judokas } \\
\qquad(n=35)\end{array}$} & \multirow{2}{*}{$\begin{array}{l}\mathrm{VO}_{2 \text { max }} \text { was measured directly during } \\
\text { maximal graded exercise lower-body } \\
\text { and upper-body cycle ergometer test. }\end{array}$} & Lower-body = $47.59 \pm 9.51$ \\
\hline & & & Upper-body $=39.44 \pm 7.87$ \\
\hline \multirow{2}{*}{ Julio et al. [24] } & \multirow{2}{*}{ Adult male judokas $(n=11)$} & \multirow{2}{*}{$\begin{array}{l}\mathrm{VO}_{2 \max } \text { was measured directly during a } \\
\text { maximal graded exercise upper-body } \\
\text { and lower-body cycle ergometer test. }\end{array}$} & Upper-body $=37.70 \pm 3.83$ \\
\hline & & & Lower-body $=50.17 \pm 6.21$ \\
\hline \multirow{3}{*}{$\begin{array}{l}\text { Shiroma et al. } \\
{[25]}\end{array}$} & \multirow{3}{*}{ Male adult judokas $(n=12)$} & \multirow{3}{*}{$\begin{array}{c}\mathrm{VO}_{2 \max } \text { was measured directly during a } \\
\text { maximal graded exercise upper-body } \\
\text { and lower-body cycle ergometer test } \\
\text { and judo-specific test. }\end{array}$} & Upper-body $=37.03 \pm 7.16$ \\
\hline & & & Lower-body $=44.78 \pm 5.98$ \\
\hline & & & Judo-specific $=46.04 \pm 5.34$ \\
\hline $\begin{array}{l}\text { Sterkowicz } \\
\text { et al. [26] }\end{array}$ & Senior judokas $(n=15)$ & $\begin{array}{l}\mathrm{VO}_{2 \max } \text { was measured directly during a } \\
\text { maximal graded exercise treadmill test. }\end{array}$ & $50.1 \pm 6.48$ \\
\hline \multirow{3}{*}{$\begin{array}{l}\text { Franchini et al. } \\
\text { [27] }\end{array}$} & \multirow{3}{*}{$\begin{array}{l}\text { Adult male judokas (national and international } \\
\text { levels, } n=5 \text {; state level, } n=7 \text {; city level, } n=5 \text { ) }\end{array}$} & \multirow{3}{*}{$\begin{array}{l}\mathrm{VO}_{2 \max } \text { was measured directly during a } \\
\text { maximal graded exercise treadmill test. }\end{array}$} & $\begin{array}{c}\text { National/International }= \\
63.0 \pm 10.3\end{array}$ \\
\hline & & & State $=62.9 \pm 9.3$ \\
\hline & & & City $=64.9 \pm 5.5$ \\
\hline \multirow{2}{*}{$\begin{array}{l}\text { Franchini et al. } \\
\text { [28] }\end{array}$} & \multirow{2}{*}{$\begin{array}{c}\text { Elite }(n=15) \text { and non-elite }(n=31) \text { Brazilian } \\
\text { male adult judokas }\end{array}$} & \multirow{2}{*}{$\begin{array}{l}\mathrm{VO}_{2 \max } \text { was measured directly during a } \\
\text { maximal graded exercise treadmill test. }\end{array}$} & Elite $=58.13 \pm 10.83$ \\
\hline & & & Non-elite $=63.28 \pm 10.55$ \\
\hline Sbriccoli et al. & Italian Olympic team (males, $n=6$; females, & $\mathrm{VO}_{2 \max }$ was measured directly during a & Males $=47.3 \pm 10.9$ \\
\hline [29] & & & Females $=52.9 \pm 4.4$ \\
\hline & & & $62.6 \pm 7.4$ \\
\hline Franchini et al. & $\begin{array}{l}\text { Male adult black belt judokas (study 1, } n=16 \\
\text { study } 2 \text {; }\end{array}$ & $\begin{array}{l}\mathrm{VO}_{2 \max } \text { was measured directly during a } \\
\text { maximal graded exercise treadmill test. }\end{array}$ & $58.8 \pm 7.8$ \\
\hline & & & $59.5 \pm 6.3$ \\
\hline Trivic et al. [31] & Active male judokas $(n=20)$ & $\begin{array}{l}\mathrm{VO}_{2 \max } \text { was measured directly during a } \\
\text { maximal graded exercise treadmill test. }\end{array}$ & $52.6 \pm 2.5$ \\
\hline $\begin{array}{l}\text { Santos et al. } \\
{[32]}\end{array}$ & Highly-trained male judokas $(n=8)$ & $\begin{array}{l}\mathrm{VO}_{2 \max } \text { was measured directly during a } \\
\text { maximal graded exercise treadmill test. }\end{array}$ & $58.3 \pm 4.4$ \\
\hline $\begin{array}{l}\text { Santos et al. } \\
{[33]}\end{array}$ & Highly-trained male judokas $(n=8)$ & $\begin{array}{l}\mathrm{VO}_{2 \max } \text { was measured directly during a } \\
\text { maximal graded exercise treadmill test. }\end{array}$ & $52.8 \pm 7.9$ \\
\hline $\begin{array}{c}\text { Franchini et al. } \\
\text { [34] }\end{array}$ & Male adult black belt judokas $(n=14)$ & $\begin{array}{l}\mathrm{VO}_{2 \max } \text { was measured directly during a } \\
\text { maximal graded exercise treadmill test. }\end{array}$ & $56.42 \pm 3.61$ \\
\hline $\begin{array}{l}\text { Santos et al. } \\
{[35]}\end{array}$ & Highly-trained female judokas $(n=8)$ & $\begin{array}{l}\mathrm{VO}_{2 \max } \text { was measured directly during a } \\
\text { maximal graded exercise treadmill test. }\end{array}$ & $40.9 \pm 7.7$ \\
\hline Radjo et al. [36] & Male judokas (younger senior, $n=8$; senior, & $\mathrm{VO}_{2 \max }$ was measured directly during a & Younger seniors $=56.5 \pm 3.1^{*}$ \\
\hline & & & Senior $=50.6 \pm 2.5$ \\
\hline Drid et al. [37] & Elite $(n=5)$ and sub-elite $(n=5)$ European & $\mathrm{VO}_{2 \max }$ was measured directly during a & Elite $=55.99 \pm 2.24$ \\
\hline & & & Sub-elite $=48.72 \pm 1.72^{\mathrm{b}}$ \\
\hline
\end{tabular}


Table 1. Cont.

\begin{tabular}{|c|c|c|c|}
\hline Study & Group Characteristics & Test and Measurement Characteristics & $\begin{array}{c}\mathrm{VO}_{2 \max }\left(\mathrm{mL} \cdot \mathrm{kg}^{-1} \cdot \mathrm{min}^{-1} \text { Unless }\right. \\
\text { Specified Otherwise })\end{array}$ \\
\hline \multirow{2}{*}{$\begin{array}{l}\text { Schwartz et al. } \\
{[38]}\end{array}$} & \multirow{2}{*}{ Adult judo practitioners $(n=180)$} & $\mathrm{VO}_{2 \max }$ was estimated via Queen's & \multirow{2}{*}{$52.40 \pm 8.29$} \\
\hline & & college step test. & \\
\hline $\begin{array}{l}\text { Coswig et al. } \\
\text { [39] }\end{array}$ & $\begin{array}{c}\text { Male adult judokas }(n=16) \text {, regional or higher } \\
\text { level }\end{array}$ & $\begin{array}{l}\mathrm{VO}_{2 \max } \text { was estimated based on a } \\
\text { maximal graded exercise treadmill test. }\end{array}$ & $50.1 \pm 7.9$ \\
\hline \multirow{2}{*}{$\begin{array}{l}\text { Franchini et al. } \\
\text { [40] }\end{array}$} & \multirow{2}{*}{$\begin{array}{l}\text { Brazilian senior national team } \mathrm{A}(n=7) \text { and } \\
\text { reserves }(n=15)\end{array}$} & \multirow{2}{*}{$\begin{array}{l}\mathrm{VO}_{2 \max } \text { was estimated based on the } \\
\text { Cooper test. }\end{array}$} & Team $\mathrm{A}=48.3 \pm 8.1$ \\
\hline & & & Reserves $=49.6 \pm 5.5$ \\
\hline \multirow{3}{*}{ Drid et al. [41] } & \multirow{3}{*}{$\begin{array}{l}\text { Serbian female adult national team ( } n=8 \\
\text { international medal winners; } n=8 \text { non-medal } \\
\text { winners in international competitions) }\end{array}$} & \multirow{3}{*}{$\begin{array}{l}\mathrm{VO}_{2 \max } \text { was estimated via multistage } \\
20-\mathrm{m} \text { shuttle run test. }\end{array}$} & All $=45.8 \pm 5.2$ \\
\hline & & & $\begin{array}{l}\text { International medal winners }= \\
\quad 49.5 \pm 4.0\end{array}$ \\
\hline & & & $\begin{array}{c}\text { Non-medal winners in } \\
\text { international competitions }= \\
42.0 \pm 3.0\end{array}$ \\
\hline \multirow{2}{*}{$\underset{[42]}{\text { Almansba et al. }}$} & Algerian Olympic judo team, males $(n=7)$ & \multirow{2}{*}{$\begin{array}{l}\mathrm{VO}_{2 \max } \text { was estimated based on the } \\
\text { PWC }_{170} \text { test. }\end{array}$} & $50.6 \pm 9.3$ \\
\hline & Females $(n=6)$ & & $50.7 \pm 6.0$ \\
\hline \multirow{6}{*}{ Almansba et al. } & \multirow{6}{*}{$\begin{array}{c}\text { Algerian adult judokas, males }(n=15) \\
\text { Departamental level }(n=7) \\
\text { Inter-regional level }(n=8)\end{array}$} & \multirow{6}{*}{$\begin{array}{l}\mathrm{VO}_{2 \max } \text { was estimated via multistage } \\
20-\mathrm{m} \text { shuttle run test. }\end{array}$} & $\mathrm{D}, \mathrm{G}=51.64 \pm 0.31$ \\
\hline & & & $\mathrm{D}, \mathrm{ST}=52.38 \pm 0.31$ \\
\hline & & & $\mathrm{D}, \mathrm{CP}=51.90 \pm 0.31$ \\
\hline & & & $\mathrm{I}, \mathrm{G}=51.83 \pm 0.29$ \\
\hline & & & $\mathrm{I}, \mathrm{ST}=52.39 \pm 0.29$ \\
\hline & & & $\mathrm{I}, \mathrm{CP}=51.85 \pm 0.30$ \\
\hline $\begin{array}{l}\text { Bermi and Ali } \\
\text { [44] }\end{array}$ & Iranian female adult judo team $(n=7)$ & $\begin{array}{c}\mathrm{VO}_{2 \max } \text { was estimated based on the } \\
\text { Bruce test. }\end{array}$ & $46.9 \pm 5.32$ \\
\hline \multirow{8}{*}{$\begin{array}{l}\text { Tartibian et al. } \\
{[45]}\end{array}$} & \multirow{8}{*}{$\begin{array}{l}\text { Healthy elite male adult judokas (Control } \\
\text { group, } n=12 \text {; Exercise group, } n=12 \text { ) }\end{array}$} & \multirow{8}{*}{$\begin{array}{l}\mathrm{VO}_{2 \max } \text { was measured during the Bruce } \\
\text { test. }\end{array}$} & Control \\
\hline & & & Pre $=51.65 \pm 2.14$ \\
\hline & & & Post $=51.05 \pm 2.35$ \\
\hline & & & $\begin{array}{l}\text { Recovery ( } 1 \text { weeks after training } \\
\text { finished })=51.05 \pm 2.35\end{array}$ \\
\hline & & & Exercise group \\
\hline & & & Pre $=52.24 \pm 2.48$ \\
\hline & & & Post $=53.67 \pm 3.25^{\mathrm{a}, \mathrm{b}}$ \\
\hline & & & $\begin{array}{l}\text { Recovery ( } 1 \text { week after training } \\
\text { finished })=53.65 \pm 3.25^{\mathrm{b}}\end{array}$ \\
\hline $\begin{array}{c}\text { Garbouj et al. } \\
{[46]}\end{array}$ & $\begin{array}{c}\text { Adult female judokas ( } n=17, n=10 \text { national } \\
\text { level, } n=7 \text { regional level) }\end{array}$ & $\begin{array}{l}\mathrm{VO}_{2 \max } \text { was estimated via multistage } \\
20-\mathrm{m} \text { shuttle run test. }\end{array}$ & $42.5 \pm 7.8$ \\
\hline $\begin{array}{l}\text { Morales et al. } \\
{[47]}\end{array}$ & $\begin{array}{l}\text { International-standard judokas ( } 7 \text { males and } \\
\text { four females) }\end{array}$ & $\begin{array}{l}\mathrm{VO}_{2 \max } \text { was measured directly during } \\
\text { maximal graded exercise cycle } \\
\text { ergometer test. }\end{array}$ & $53.17 \pm 5.6$ \\
\hline Arazi et al. [48] & $\begin{array}{c}\text { Senior Iranian male judokas ( } 3^{\text {rd }} \text { kyu to 3rd } \\
\text { DAN, } n=50 \text { ) }\end{array}$ & $\begin{array}{l}\mathrm{VO}_{2 \max } \text { was estimated based on the } \\
\text { Cooper test. }\end{array}$ & $54.4 \pm 6.4$ \\
\hline \multirow{2}{*}{$\begin{array}{l}\text { Quintero et al. } \\
\text { [49] }\end{array}$} & \multirow{2}{*}{$\begin{array}{l}\text { Colombian international level judokas (males, } \\
\qquad n=7 \text {; females, } n=8 \text { ) }\end{array}$} & \multirow{2}{*}{$\begin{array}{l}\mathrm{VO}_{2 \max } \text { was estimated via multistage } \\
20-\mathrm{m} \text { shuttle run test. }\end{array}$} & Males $=51.2 \pm 5.6$ \\
\hline & & & Females $=43.6 \pm 4.3$ \\
\hline
\end{tabular}

Legend: $\mathrm{VO}_{2 \max }$, maximal oxygen consumption; ${ }^{*}$, difference between groups $(p<0.05)$; D, departmental; I, inter-regional; $\mathrm{G}$, general preparatory period; ST, special training period; $\mathrm{CP}$, competitive period; a, different from pre- for the same group $(p<0.05)$; $b$, different from control group for the same time-point $(p<0.05)$.

Borkowski et al. [14] compared $\mathrm{VO}_{2 \max }$ among weight categories. Male heavyweights presented lower values compared with athletes from all other weight classes, while $\mathrm{VO}_{2 \max }$ among female athletes was similar regardless of weight class.

\subsection{Respiratory Function}

One study [43] reported that recreational judo training in female students was associated with higher values of pulmonary function tests (vital capacity, forced vital capacity (FVC), forced expiratory volume in 1st second (FEV1), and peak expiratory flow (PEF) compared to a control group of students not engaged in sports. The most significant differ- 
ence was reported for PEF (448.1 \pm 52.2 vs. $\left.370.9 \pm 75.6 \mathrm{dm}^{3} / \mathrm{min}\right)$. The shared variance between PEF and chest mobility (R\%) suggested that judo training had a positive influence on the respiratory system. Ermis et al. [50] found that elite female judokas specifically had stronger respiratory muscles compared to a sedentary female control group (maximal inspiratory mouth pressure $103.4 \pm 23.2$ vs. $81.314 .4(\mathrm{cmH} 2 \mathrm{O}), p=0.003$; maximal expiratory mouth pressure $135.7 \pm 27$ vs. $95 \pm 19.4(p<0.001))$, whereas other respiratory function tests values were found to be similar. Respiratory function and respiratory muscle strength were compared among athletes partaking in judo, Muay Thai, boxing, and taekwondo [51]. Judo and Muay Thai athletes had stronger respiratory muscles than the other two groups ( $p=0.025)$, however, pulmonary function tests values (FVC, FEV1, maximal voluntary ventilation, and PEF) did not differ significantly.

\subsection{Body Composition}

Twenty-seven studies have evaluated the effect of judo training on body composition [20,21,34,37,45,48,49,52-71] (Table 2). Andreoli et al. [69] screened karate, judo, and water polo athletes for their body composition, and found that judokas had less body fat than their water polo counterparts. However, water polo athletes were leaner, which is likely due to their overall greater height and mass. Also, judokas had less fat than their karate counterparts, while also having more muscle mass. Burdukiewicz et al. [51] assessed female athletes participating in judo and weightlifting, and found that judokas had less fat than weightlifters, but also had significantly less muscle mass $(p<0.05)$ when compared to weightlifters. In addition, judokas had significantly more fat-free mass $(p<0.05)$ in comparison to weightlifters. Busko et al. [58] found that male judokas had significantly less fat percentage and more fat-free mass than untrained students $(p<0.05)$. Socha et al. [57] showed that female judokas had significantly better body mass index compared to untrained female subjects $(p<0.01)$, while also having more muscle mass. However, judokas had more fat when compared to untrained participants. Mala et al. [60] screened judo, karate, fencing, wrestling, taekwondo, and kickboxing athletes, and found similar BMI values among all groups. Fat-free mass significantly differed between groups $(p<0.01)$, with judokas showing the greatest mean values followed by kickboxers and wrestlers. A low proportion of fat mass was detected within all groups, with no significant difference between sports. Shariat et al. [66] found that judokas had significantly higher fat mass compared to karate and taekwondo athletes $(p<0.05)$. However, judokas demonstrated a lower percentage of muscle mass compared to the other two groups $(p<0.05)$. Reale et al. [68] screened judo, wrestling, taekwondo, and boxing athletes, and found that male judokas had significantly greater body mass compared to male boxers $(p<0.05)$, but the lean mass percentage in boxers and taekwondo athletes was significantly higher than in judokas $(p<0.05)$. Consequently, fat mass differed in the same fashion across these three sports, whereas male judokas presented significantly higher BMI values than boxers $(p<0.05)$ and taekwondo athletes $(p<0.0001)$, with no significant differences in female participants.

Table 2. Body composition characteristics of judokas and judo practitioners.

\begin{tabular}{|c|c|c|c|c|c|c|c|c|c|}
\hline \multirow[t]{2}{*}{ Study } & \multirow{2}{*}{$\begin{array}{l}\text { Group } \\
\text { Charac- } \\
\text { teristics }\end{array}$} & \multirow{2}{*}{$\begin{array}{c}\text { Test and } \\
\text { Measurements } \\
\text { Characteristics }\end{array}$} & \multicolumn{7}{|c|}{ Body Composition } \\
\hline & & & $\begin{array}{c}\text { BM } \\
\text { (kg) }\end{array}$ & $\begin{array}{c}\text { BMI } \\
\left(\mathrm{kg} / \mathrm{m}^{2}\right)\end{array}$ & $\begin{array}{l}\text { FM } \\
\text { (kg) }\end{array}$ & $\begin{array}{l}\text { FM } \\
(\%)\end{array}$ & $\begin{array}{l}\text { FFM } \\
\text { (kg) }\end{array}$ & FFM (\%) & LBM (kg) \\
\hline $\begin{array}{l}\text { Andreoli } \\
\text { et al. [69] }\end{array}$ & $\begin{array}{l}\text { males } \\
n=12\end{array}$ & DEXA & $74.7 \pm 10.1$ & $24.7 \pm 2.1$ & $7.5 \pm 3.6$ & NA & NA & NA & 63.3. \pm 7.2 \\
\hline $\begin{array}{c}\text { Silva et al. } \\
\text { [70] }\end{array}$ & $\begin{array}{l}\text { males } \\
n=27\end{array}$ & DEXA & $72.8 \pm 7.1$ & $23.6 \pm 2.3$ & $8.8 \pm 2.8$ & $12.1 \pm 3.1$ & $63.4 \pm 5.7$ & NA & NA \\
\hline $\begin{array}{l}\text { Goncalves } \\
\text { et al. [71] }\end{array}$ & $\begin{array}{l}\text { males } \\
n=32\end{array}$ & DEXA & $73.4 \pm 8.3$ & NA & NA & $12.1 \pm 3.1$ & NA & $63.9 \pm 6.3$ & NA \\
\hline $\begin{array}{c}\text { Murata et al. } \\
{[52]}\end{array}$ & $\begin{array}{l}\text { males } \\
n=19\end{array}$ & DEXA & $122.7 \pm 13.1$ & $39.1 \pm 3.8$ & $34.1 \pm 8.8$ & $27.5 \pm 5.2$ & $88.6 \pm 8.0$ & NA & NA \\
\hline
\end{tabular}


Table 2. Cont.

\begin{tabular}{|c|c|c|c|c|c|c|c|c|c|}
\hline \multirow[t]{2}{*}{ Study } & \multirow{2}{*}{$\begin{array}{l}\text { Group } \\
\text { Charac- } \\
\text { teristics }\end{array}$} & \multirow{2}{*}{$\begin{array}{c}\text { Test and } \\
\text { Measurements } \\
\text { Characteristics }\end{array}$} & \multicolumn{7}{|c|}{ Body Composition } \\
\hline & & & $\begin{array}{l}\text { BM } \\
(\mathbf{k g})\end{array}$ & $\begin{array}{c}\text { BMI } \\
\left(\mathrm{kg} / \mathrm{m}^{2}\right)\end{array}$ & $\begin{array}{l}\text { FM } \\
\text { (kg) }\end{array}$ & $\begin{array}{l}\text { FM } \\
(\%)\end{array}$ & $\begin{array}{l}\text { FFM } \\
\text { (kg) }\end{array}$ & FFM (\%) & LBM (kg) \\
\hline \multirow[t]{2}{*}{$\begin{array}{l}\text { Reale et al. } \\
\text { [68] }\end{array}$} & $\begin{array}{l}\text { males } \\
n=17\end{array}$ & DEXA & $80.7 \pm 14.9$ & $26.1 \pm 3.4$ & $12.3 \pm 6.7$ & $14.5 \pm 5.7$ & NA & NA & $\begin{array}{l}64.4 \pm 9.3 \\
80.4 \pm 5.5 \\
\quad(\%)\end{array}$ \\
\hline & $\begin{array}{c}\text { females } \\
n=7\end{array}$ & & $68.1 \pm 11.4$ & $24.5 \pm 3.2$ & $17.6 \pm 7.9$ & $24.9 \pm 7.6$ & NA & NA & $\begin{array}{l}47.4 \pm 4.0 \\
70.6 \pm 7.5 \\
\quad(\%)\end{array}$ \\
\hline $\begin{array}{l}\text { Burdukiewitz } \\
\text { et al. [53] }\end{array}$ & $\begin{array}{c}\text { females } \\
n=28\end{array}$ & BIA & $62.9 \pm 18.7$ & $22.6 \pm 3.9$ & $16.6 \pm 11.3$ & $24.9 \pm 6.3$ & NA & NA & $\begin{array}{c}31.5 \pm 3.7 \\
52.1 \pm 9.0 \\
\quad(\%)\end{array}$ \\
\hline Ocak [54] & $\begin{array}{c}\text { females } \\
n=25\end{array}$ & BIA & $62.7 \pm 22.7$ & $23.1 \pm 6.2$ & NA & $17.31 \pm 1.52$ & NA & NA & NA \\
\hline $\begin{array}{l}\text { Laskowski } \\
\text { et al. [55] }\end{array}$ & $\begin{array}{l}\text { males } \\
n=11\end{array}$ & BIA & $83.7 \pm 8.3$ & $25.2 \pm 1.5$ & $8.5 \pm 3.7$ & $9.9 \pm 3.2$ & $75.2 \pm 5.6$ & NA & NA \\
\hline $\begin{array}{l}\text { Smulski } \\
\text { et al. [56] }\end{array}$ & $\begin{array}{c}\text { females } \\
n=11\end{array}$ & BIA & $65.6 \pm 12.1$ & $23.1 \pm 3.2$ & $13.6 \pm 6.6$ & $19.9 \pm 5.4$ & $52.1 \pm 6.2$ & $80.1 \pm 5.4$ & NA \\
\hline $\begin{array}{l}\text { Mala et al. } \\
\text { [20] }\end{array}$ & $\begin{array}{l}\text { males } \\
n=9\end{array}$ & BIA & $84.7 \pm 10.2$ & NA & NA & $12.9 \pm 2.8$ & $73.3 \pm 7.5$ & NA & NA \\
\hline $\begin{array}{l}\text { Tartibian } \\
\text { et al. [45] }\end{array}$ & $\begin{array}{l}\text { males } \\
n=24\end{array}$ & BIA & $77.1 \pm 7.3$ & $21.9 \pm 2.0$ & NA & $8.7 \pm 2.4$ & NA & NA & NA \\
\hline $\begin{array}{c}\text { Socha et al. } \\
\text { [57] }\end{array}$ & $\begin{array}{c}\text { females } \\
n=25\end{array}$ & BIA & $60.5 \pm 9.4$ & $22.1 \pm 2.0$ & $15.9 \pm 3.7$ & $26.1 \pm 3.2$ & $44.6 \pm 6.5$ & $73.8 \pm 3.2$ & NA \\
\hline \multirow{2}{*}{$\begin{array}{c}\text { Kujach et al. } \\
\text { [21] }\end{array}$} & $\begin{array}{l}\text { males } \\
n=6\end{array}$ & BIA & $87 \pm 11.6$ & $26.4 \pm 3.3$ & $10.2 \pm 4.9$ & $11.3 \pm 4.1$ & $76.8 \pm 7.7$ & NA & NA \\
\hline & $\begin{array}{c}\text { females } \\
n=6\end{array}$ & & $61.9 \pm 10.2$ & $23.4 \pm 2.8$ & $13.9 \pm 4.9$ & $22 \pm 4.5$ & $47.9 \pm 5.4$ & NA & NA \\
\hline $\begin{array}{c}\text { Busko et al. } \\
\text { [58] }\end{array}$ & $\begin{array}{l}\text { males } \\
n=15\end{array}$ & BIA & $80.2 \pm 15.7$ & $25.3 \pm 3.4$ & $8.7 \pm 4.2$ & $10.5 \pm 3.8$ & $71.5 \pm 12.9$ & NA & NA \\
\hline \multirow{2}{*}{$\begin{array}{c}\text { Ceylan et al. } \\
\text { [59] }\end{array}$} & $\begin{array}{l}\text { males } \\
n=7\end{array}$ & BIA & $88.1 \pm 22.8$ & $29.4 \pm 5.8$ & $12.8 \pm 7.2$ & $13.6 \pm 4.4$ & $75.3 \pm 15.7$ & NA & NA \\
\hline & $\begin{array}{c}\text { females } \\
n=10\end{array}$ & & $67.6 \pm 19.8$ & $25.4 \pm 5.7$ & $15.5 \pm 8.9$ & $21.6 \pm 5.8$ & $52.1 \pm 11.1$ & NA & NA \\
\hline $\begin{array}{l}\text { Mala et al. } \\
\text { [60] }\end{array}$ & $\begin{array}{l}\text { males } \\
n=19\end{array}$ & BIA & $83.3 \pm 11.6$ & $24.6 \pm 2.4$ & NA & $9.8 \pm 3.7$ & $74.5 \pm 9.9$ & NA & NA \\
\hline $\begin{array}{l}\text { Franchini } \\
\text { et al. [34] }\end{array}$ & $\begin{array}{l}\text { males } \\
n=22\end{array}$ & Skinfold & $88.5 \pm 20.0$ & NA & NA & $10.7 \pm 7.0$ & NA & NA & NA \\
\hline \multirow[t]{2}{*}{$\begin{array}{l}\text { Quintero } \\
\text { et al. [49] }\end{array}$} & $\begin{array}{l}\text { males } \\
n=7\end{array}$ & Skinfold & $73.1 \pm 11$ & NA & NA & $15.2 \pm 5.8$ & NA & NA & $57.9 \pm 7$ \\
\hline & $\begin{array}{c}\text { females } \\
n=8\end{array}$ & & $61 \pm 14.4$ & NA & NA & $17.3 \pm 4.94$ & NA & NA & $44.2 \pm 9.7$ \\
\hline \multirow[t]{2}{*}{$\begin{array}{l}\text { Franchini } \\
\text { et al. [61] }\end{array}$} & \multirow{2}{*}{$\begin{array}{c}\text { males } \\
n=23 \\
\text { females } \\
n=30\end{array}$} & \multirow[t]{2}{*}{ Skinfold } & $82.5 \pm 5.3$ & NA & $8.4 \pm 2.8$ & $9.3 \pm 2.2$ & NA & NA & $\begin{array}{c}43.3 \pm 1.9 \\
51.7 \pm 1.8 \\
\quad(\%)\end{array}$ \\
\hline & & & $67.7 \pm 4.5$ & NA & $16.1 \pm 2.4$ & $21.9 \pm 1.4$ & NA & NA & $\begin{array}{l}28.1 \pm 1.6 \\
41.1 \pm 1.5 \\
\quad(\%)\end{array}$ \\
\hline \multirow{2}{*}{$\begin{array}{l}\text { Sterkowitz- } \\
\text { Przybycien } \\
\text { et al. [62] }\end{array}$} & $\begin{array}{l}\text { males } \\
n=22\end{array}$ & \multirow[t]{2}{*}{ Skinfold } & $87.4 \pm 24.9$ & $26.8 \pm 5.20$ & $13.3 \pm 8.5$ & $14.2 \pm 4.2$ & $74.0 \pm 16.6$ & $85.7 \pm 4.2$ & NA \\
\hline & $\begin{array}{c}\text { females } \\
n=12\end{array}$ & & $74.8 \pm 23.9$ & $25.7 \pm 5.5$ & $18.0 \pm 8.9$ & $23.2 \pm 3.6$ & $56.8 \pm 15.2$ & $76.7 \pm 3.6$ & NA \\
\hline $\begin{array}{c}\text { Katralli et al. } \\
\text { [63] }\end{array}$ & $\begin{array}{c}\text { females } \\
n=31\end{array}$ & Skinfold & $60.7 \pm 10.0$ & $23.2 \pm 3.3$ & NA & $12.8 \pm 6.0$ & NA & NA & NA \\
\hline $\begin{array}{l}\text { Drid et al. } \\
\text { [37] }\end{array}$ & $\begin{array}{l}\text { males } \\
n=10\end{array}$ & Skinfold & $100.5 \pm 0.9$ & NA & NA & $15.6 \pm 0.64$ & NA & NA & NA \\
\hline \multirow[t]{2}{*}{$\begin{array}{c}\text { Casals et al. } \\
{[64]}\end{array}$} & \multirow{2}{*}{$\begin{array}{c}\text { males } \\
n=20 \\
\text { females } \\
n=29\end{array}$} & \multirow[t]{2}{*}{ Skinfold } & $79.3 \pm 29.9$ & NA & NA & $7.5 \pm 5.41$ & NA & NA & $\begin{array}{c}52.3 \pm 3.9 \\
(\%)\end{array}$ \\
\hline & & & $66.1 \pm 20$ & NA & NA & $20.9 \pm 10.0$ & NA & NA & $44 \underset{(\%)}{ \pm 10.3}$ \\
\hline $\begin{array}{c}\text { Arazi et al. } \\
{[48]}\end{array}$ & $\begin{array}{l}\text { males } \\
n=50\end{array}$ & Skinfold & $70.0 \pm 8.2$ & $21.3 \pm 2.8$ & NA & $11.4 \pm 2.2$ & $62.7 \pm 6.17$ & NA & NA \\
\hline $\begin{array}{c}\text { Tavares } \\
\text { Junior et al. } \\
{[65]}\end{array}$ & $\begin{array}{l}\text { males } \\
n=19\end{array}$ & Skinfold & $87.1 \pm 21.4$ & NA & $15.3 \pm 7.3$ & $16.8 \pm 5.6$ & $71.4 \pm 12.4$ & NA & $21.7 \pm 2.3$ \\
\hline $\begin{array}{c}\text { Shariat et al. } \\
{[66]}\end{array}$ & $\begin{array}{l}\text { males } \\
n=42\end{array}$ & $\begin{array}{l}\text { Medical scale } \\
\text { Skinfold } \\
\text { Equations (FM) }\end{array}$ & $73.8 \pm 14.9$ & $23.3 \pm 3.2$ & $10.1 \pm 3.6$ & NA & NA & NA & $\begin{array}{c}63.7 \pm 11.9 \\
86.6 \pm 2.4 \\
(\%)\end{array}$ \\
\hline
\end{tabular}


Table 2. Cont.

\begin{tabular}{|c|c|c|c|c|c|c|c|c|c|}
\hline \multirow[t]{2}{*}{ Study } & \multirow{2}{*}{$\begin{array}{l}\text { Group } \\
\text { Charac- } \\
\text { teristics }\end{array}$} & \multirow{2}{*}{$\begin{array}{c}\text { Test and } \\
\text { Measurements } \\
\text { Characteristics }\end{array}$} & \multicolumn{7}{|c|}{ Body Composition } \\
\hline & & & $\begin{array}{l}\text { BM } \\
\text { (kg) }\end{array}$ & $\underset{\left(\mathrm{kg} / \mathrm{m}^{2}\right)}{\mathrm{BMI}}$ & $\begin{array}{l}\text { FM } \\
\text { (kg) }\end{array}$ & $\begin{array}{l}\text { FM } \\
(\%)\end{array}$ & $\begin{array}{l}\text { FFM } \\
\text { (kg) }\end{array}$ & FFM (\%) & LBM (kg) \\
\hline $\begin{array}{c}\text { Kubo et al. } \\
\text { [67] }\end{array}$ & $\begin{array}{l}\text { males } \\
n=69\end{array}$ & USI & $86.5 \pm 20$ & NA & NA & NA & $70.4 \pm 10$ & NA & NA \\
\hline
\end{tabular}

Legend: BM, body mass; BMI, body mass index; FM, fat mass; FFM, fat-free mass; LBM, lean body mass; \%, lean body mass expressed as percentage; F, females; M, males; NA, not available; S, senior athletes; J, junior athletes; BIA, bioelectric impedance; DEXA, dual X-ray absorptiometry; USI, ultrasound imaging; NT, national team judokas; VT, university varsity team; JT, junior varsity team.

\subsection{Arterial Hypertension}

Casals et al. [64] revealed that 50\% of male judokas had high blood pressure (BP), $30 \%$ of male judokas were diagnosed with stage I hypertension, and $17 \%$ of females had high blood pressure. Mean resting BP values were similar when compared among weight categories in males $(-60,-66 \mathrm{~kg}: 120 \pm 8.94 \mathrm{mmHg}$ vs. $-73,-81,-90 \mathrm{~kg}$ : $126 \pm 18.50 \mathrm{mmHg}$ vs. $-100,+100 \mathrm{~kg}: 133 \pm 14.83 \mathrm{mmHg}, p=0.397)$. Karagounis et al. [72] investigated diastolic diameter and mean blood flow velocity of six conduit arteries of the upper and lower limbs, and found a significantly increase $(p<0.05)$ in the judokas. The male judokas had mean systolic and diastolic values of $119 \mathrm{mmHg}$ and $73 \mathrm{mmHg}$, respectively, and the female judo athlete group had values of $121 \mathrm{mmHg}$ and $72 \mathrm{mmHg}$, respectively. Whyte et al. [73] found that all athletes included in their study had normal diastolic and systolic function.

\subsection{Cardiac Remodeling}

Five studies have evaluated the effect of judo participation on heart size [73-77]. In a study done by Pelliccia et al. [74] conducted on 600 females, athletes had $6 \%$ larger left ventricular (LV) end-diastolic dimensions and $14 \%$ greater maximal wall thickness (MWT) than sedentary controls. Judokas had a mean MWT of $8.2 \pm 0.8$ and left ventricular mass (LVM) index $81 \pm 17 \mathrm{~g} / \mathrm{m}^{2}$. Date et al. [75] found that LVM and LV wall thickness (LVWT) of marathon runners and judo practitioners were significantly greater than those of controls (LVM $318.3 \pm 48$ vs. $496.2 \pm 114.8$ vs. $189.7 \pm 36$ g, respectively $p<0.0001$; LVWT $21.9 \pm 1.9$ vs. $25.5 \pm 3.5$ vs. $16.2 \pm 2.7 \mathrm{~mm}, p<0.001)$. The LV end-diastolic dimension index was significantly more significant in the marathon group and smaller in the judokas compared to the control $\left(31.2 \pm 2.1 \mathrm{vs} .22 .7 \pm 2.9\right.$ vs. $\left.26.9 \pm 2.2 \mathrm{~mm} / \mathrm{m}^{2}\right)$. Plasma brain natriuretic peptide (NP) concentrations were higher in both the judo and marathon groups than in controls, and positively correlated with LVM.

Sun et al. [76] included 13 male judokas, which all had increased LVM and LVM index (LVMI), associated with an increased left ventricular internal diameter (LVIDd). In six $(46 \%)$ of the males, LVIDd was above $60 \mathrm{~mm}$ with LVM $\geq 274 \mathrm{~g}$. In female judokas $(n=7)$, left ventricular (LV) geometry was normal (LVMI $74.7 \pm 13.3 \mathrm{~g} / \mathrm{m}^{2}$ ), with increased LVIDd (58 $\mathrm{mm})$ and LVM $(224 \mathrm{~g})$ in only one.

Whyte et al. [73] noted that judokas exhibited significantly increased LV wall thickness and LVM (males 264 vs. 191 g, females LVM 192 vs. 132 g.), with normal LVIDd and preserved systolic and diastolic function compared to sex-matched controls. Although significantly increased, wall thickness measures were within reference values for all judo athletes.

Whyte et al. [77] found that mean interventricular septal wall thickness (IVSd) was $10.9 \mathrm{~mm}$, mean posterior wall thickness (PWd) was $10.4 \mathrm{~mm}$, and left ventricular mass (LVM) was $259 \pm 64 \mathrm{~g}$ in 22 male judo athletes. In 20 female judokas, mean IVSd was $9 \mathrm{~mm}$, PWd $8.9 \mathrm{~mm}$, and LVM $190 \pm 38 \mathrm{~g}$. The LVM and LV wall thickness of marathon runners and judo athletes were significantly greater than those of controls. Two male judokas had a MWT of $14 \mathrm{~mm}$ and LVM of 370, $375 \mathrm{~g}$, respectively. 


\subsection{Blood Lipid Profile}

Four articles have evaluated the effect of judo training on blood lipid profile $[17,52,64,78]$. Degoutte et al. [17] evaluated lipid and lipoprotein levels at rest, $3 \mathrm{~min}, 60 \mathrm{~min}$, and $24 \mathrm{~h}$ after a 5 min judo match. Total cholesterol levels were: $4.5 ; 4.76 ; 4.5 ; 4.22 ; \mathrm{mmol} / \mathrm{L}$, respectively, and low-density lipoprotein (LDL) levels were: $2.9 ; 2.9 ; 2.88 ; 2.73 \mathrm{mmol} / \mathrm{L}$, respectively. Triglyceride, free fatty acid (FFA), and glycerol levels had risen significantly $3 \mathrm{~min}$ after the match, but returned to baseline values within $24 \mathrm{~h}$. High-density lipoprotein (HDL) levels were also significantly increased three minutes after the exercise, followed by a gradual decrease, reaching a level below the baseline after $24 \mathrm{~h}$. Low HDL-cholesterol levels were detected in $45 \%$ of males and $34 \%$ of female judokas [64]. HDL-cholesterol was also significantly impaired in heavier weight categories for males $(p=0.015)$, but not for females. Within a male group, $15 \%$ of participants had high triglyceride levels, and $10 \%$ had high LDL values, whereas two participants ( $4 \%$ of the sample) had altered glycaemia. The study from Murata et al. [52] showed that heavyweight judokas had a higher body fat percentage. Still, their cardiometabolic risk assessed using biochemical parameters was not significantly higher compared to heavyweight football athletes. Blood biochemical variables between heavyweight (judo and American football) and non-heavyweight group athletes (rugby) were significantly higher compared to the non-heavyweight group (ALT 38 vs. $20 ; p<0.01, \gamma$-GT 32 vs. $23 ; p<0.01$, uric acid 6.9 vs. $6.2 ; p=0.048$, glucose 85 vs. $80 ; p=0.012$, insulin 8.4 vs. 5.3; $p=0.011$, and HOMA-IR 1.72). Turkmen [78] found that judokas had significantly higher insulin ( 7 vs. $5 ; p=0.028)$, and similar total cholesterol (160 vs. $156 \mathrm{mg} / \mathrm{dL} ; p=0.959), \mathrm{F}$ (108 vs. $98 \mathrm{mg} / \mathrm{dL} ; p=0.988)$, and leptin ( 2 vs. $1.8 \mathrm{ng} / \mathrm{mL}$; $p=0.564$ ) when compared to cyclists. However, cyclists had similar LDL ( $92 \mathrm{vs.} 89 \mathrm{mg} / \mathrm{dL}$; $p=0.679$ ), and significantly higher glucose (92 vs. $83 \mathrm{mg} / \mathrm{dL} ; p=0.001)$ and HDL (55 vs. $49 \mathrm{mg} / \mathrm{dL} ; p=0.046)$.

\subsection{Energy and Bone Metabolism}

Male judokas demonstrated lower resting leptin values, but similar cholesterol, glucose, and triglycerides [79]. However, when compared to elite cyclists, judokas had greater glucose, insulin, and lower HDL cholesterol [78]. Interestingly, heavyweight male judokas had comparable glucose, insulin, and HOMA-IR (homeostatic model assessment of insulin resistance) values when compared to heavyweight football and rugby athletes [52]. In the limited data available for female athletes, extended duration judo training ( $\geq 4$ weeks) appears to result in lower leptin values in both male and female athletes $[54,80]$, with no changes in insulin [80]. Following seven days prior to competition, male and female judokas who engaged in weight cycling were shown to decrease leptin and insulin values, whereas weight cyclers and female athletes not involved in weight cycling demonstrated increased C-terminal telopeptide of type I collagen (CTx), a marker of bone resorption [81,82]. Despite this potential for short-term shifts in bone metabolism in women and those engaged in weight cycling, the judokas had greater bone uncoupling index values, indicating bone formation compared to healthy controls [82].

\subsection{Bone Mineral Density}

Five studies examined either bone mineral density (BMD) or bone mineral content (BMC) among judokas [69,82-85] (Table 3). In all included studies, dual-energy X-ray absorptiometry (DEXA) technology was used to assess bone health. 
Table 3. Bone mineral density characteristics of judokas.

\begin{tabular}{|c|c|c|c|c|c|c|}
\hline Study & $\begin{array}{l}\text { Group } \\
\text { Charac- } \\
\text { teristics }\end{array}$ & Method & \multicolumn{3}{|c|}{$\operatorname{BMD}\left(\mathrm{g} / \mathrm{cm}^{2}\right)$} & Main Results \\
\hline \multirow{2}{*}{$\begin{array}{l}\text { Matsumoto et al. } \\
{[83]}\end{array}$} & \multirow{2}{*}{$\begin{array}{c}\text { males } \\
n=14 \\
\text { females } \\
n=23\end{array}$} & \multirow{2}{*}{ DEXA } & \multicolumn{3}{|c|}{ Total $=1.23 \pm 0.02$} & $\begin{array}{l}\text { This study investigated total BMD in male and female judokas, } \\
\text { long-distance runners, and swimmers. Results showed that both }\end{array}$ \\
\hline & & & \multicolumn{3}{|c|}{ Total $=1.14 \pm 0.2$} & $\begin{array}{l}\text { male and female judokas presented significantly higher values } \\
(p<0.001) \text { than males and females practicing the other two sports. }\end{array}$ \\
\hline \multirow{2}{*}{ Andreoli et al. [69] } & \multirow{2}{*}{$\begin{array}{l}\text { males } \\
n=21\end{array}$} & \multirow{2}{*}{ DEXA } & \multicolumn{3}{|c|}{$\begin{array}{c}\text { Total }=1.40 \pm 0.06 \\
\text { BMC }(\mathrm{kg})=3.84 \pm 0.4 \\
\text { Arms }=1.18 \pm 0.06 \\
\text { Legs }=1.55 \pm 0.07\end{array}$} & $\begin{array}{l}\text { Total and regional BMD among judokas, water polo, karate athletes, } \\
\text { and control groups were investigated. Judokas demonstrated } \\
\text { significantly higher total BMD than water polo athletes and a } \\
\text { control group }(p<0.05) \text {. Also, BMC was higher in judokas only }\end{array}$ \\
\hline & & & \multicolumn{3}{|c|}{ Trunk $=1.19 \pm 0.06$} & $\begin{array}{c}\text { control group }(p<0.05) \text {. Arm BMD measured in judokas was } \\
\text { markedly higher than other groups }(p<0.05) \text {, while no differences } \\
\text { were shown for leg BMD. }\end{array}$ \\
\hline \multirow{2}{*}{ Prouteau et al. [82] } & \multirow{2}{*}{$\begin{array}{c}\text { males } \\
n=22 \\
\text { females } \\
n=26\end{array}$} & \multirow{2}{*}{ DEXA } & Total $=1.40 \pm 0.1$ & $\begin{array}{c}\text { Lumbar }= \\
1.28 \pm 0.13\end{array}$ & Hip $=1.29 \pm 0.11$ & \multirow{2}{*}{$\begin{array}{l}\text { Insignificantly higher lumbar, hip, and total BMD values were } \\
\text { observed in males compared to female judokas. }\end{array}$} \\
\hline & & & $\begin{array}{c}\text { Total }= \\
1.21 \pm 0.07\end{array}$ & $\begin{array}{l}\text { Lumbar }= \\
1.14 \pm 0.08\end{array}$ & Hip $=1.10 \pm 0.11$ & \\
\hline \multirow[t]{2}{*}{ Bozkurt [84] } & \multirow[t]{2}{*}{$\begin{array}{l}\text { males } \\
n=10\end{array}$} & \multirow[t]{2}{*}{ DEXA } & \multirow{2}{*}{\multicolumn{3}{|c|}{ Wards triangle $=1.075 \pm 0.151$}} & \multirow{2}{*}{$\begin{array}{c}\text { The study investigated regional BMD in male judokas, wrestlers, } \\
\text { taekwondo athletes, and runners. Neck BMD values in judokas } \\
\text { were similar to those of wrestlers but slightly more excellent than } \\
\text { runners and taekwondo athletes. The trochanter, intertrochanter, } \\
\text { and Ward's triangle BMD were not significantly different among } \\
\text { mentioned athletes }(p>0.05) \text {. }\end{array}$} \\
\hline & & & & & & \\
\hline Bozkurt [85] & $\begin{array}{l}\text { males } \\
n=10\end{array}$ & DEXA & \multicolumn{3}{|c|}{$\begin{aligned} \mathrm{L} 1 & =1.043 \pm 0.124 \\
\mathrm{~L} 2 & =1.132 \pm 0.108 \\
\mathrm{~L} 3 & =1.162 \pm 0.115 \\
\mathrm{~L} 4 & =1.182 \pm 0.143 \\
\mathrm{~L} 1-\mathrm{L} 4 & =1.134 \pm 0.116\end{aligned}$} & $\begin{array}{l}\text { The study investigated regional BMD in male judokas, wrestlers, } \\
\text { taekwondo athletes, and runners. Judokas demonstrated } \\
\text { significantly higher BMD for L1 }(p<0.05) \text {, along with greater values } \\
\text { in L2, L3, and L4 compared to runners }(p<0.001) \text {. }\end{array}$ \\
\hline
\end{tabular}

Legend: $n$, number of participants; DEXA, dual-energy X-ray absorptiometry; BMD, bone mineral density; BMC, bone mineral content.

\subsection{Endocrine Responses}

The majority of studies examining endocrine responses to judo-specific training activity have focused mainly on testosterone and cortisol responses, and to a lesser extent, growth hormone and catecholamine responses. With respect to the former, there appears to be some variation in responses among judokas following short and extended period exercise and judo training interventions. Extended periods of judo training ( $\geq 4$ weeks) do not appear to influence cortisol responses [86], whereas the effects of training on testosterone responses [86] and the ratio of testosterone to cortisol [86,87] are mixed. One study found that female athletes demonstrated increased cortisol during the seven-day period prior to the competition when compared to controls [88]. Similarly, mixed responses to judo competition or a single training session have been observed, with studies indicating either increased testosterone $[13,89]$, increased cortisol $[89,90]$, or no change in either hormone $[90,91]$. Only one of the reviewed studies examined the influence of a judo competition on the ratio of testosterone to cortisol in judokas, and no change was observed [91]. More limited research has examined the effects of a single judo match and caloric restriction on testosterone, and cortisol responses in judokas. Preliminary evidence indicates that an acute caloric restriction and weight cycling in elite judo athletes may be associated with increased cortisol [82]. The effects of judo training on catecholamine responses are less well characterized, with only one investigation having been conducted among the studies reviewed here. Results from this study indicate that catecholamine responses to all-out exercise in collegiate judokas are influenced by the type of training implemented (resistance vs. interval training vs. only judo training) [88]. Differences in endocrine responses between studies may also be the result of variability in programmed activities within a given training session. Observations that randori training (free practice) elicits greater testosterone and cortisol responses when compared to kata (forms practice) lend support to this notion [89], where it is thought that the more aggressive behaviors associated with randori may precipitate a more robust hormonal response.

\subsection{Immune Responses and Inflammation}

Perturbations to both inflammatory and anti-inflammatory cytokines have been reported in response to a single judo training session, simulated judo competitions, and multi-day judo training sessions $[55,89,91,92]$. A single judo training session has been 
shown to result in increased neutrophil activation and cell count, as well as increased neutrophil oxidative burst activity, phagocytic activity, and reactive oxygen species (ROS) production [92]. Elevations in interleukin-6 (IL-6) have been reported over the course of simulated judo competition, whereas monocyte chemoattractant protein-1 (MCP-1), interleukin-10 (IL-10), and tumor necrosis factor-alpha (TNF $\alpha$ ) remain unchanged [91]. In contrast, IL-6 and IL-10 exhibit similar, but variable, profiles following a three-day training session, increasing immediately post-training, and decreasing below baseline values 12-h after training [55]. A similar response was observed for interleukin-1 beta (IL-1beta), whereas TNF $\alpha$ decreased following training. Less is known regarding the effects of longer-term training interventions on immune and inflammatory responses. Only two of the reviewed studies examined immune responses to long-term training interventions ( $>12$ weeks). Both of these studies indicate that extended periods of judo training do not influence immunoglobulins A, G, or M [86,93], which appears to be in line with the evidence indicating only transient changes in immunoglobulin A during a three-week training period followed by a two-week taper [89]. Finally, though judokas are reported to have lower resting MCP-1 values compared to untrained controls, they exhibit similar resting values for IL- 6 and tumor necrosis factor-alpha (TNF $\alpha$ ), suggesting that judo training does not have a profound impact on resting cytokine values [79].

\subsection{Muscle Damage and Oxidative Stress}

The strenuous physical requirements of judo training may result in elevated markers of muscle damage and oxidative stress. Perhaps not surprisingly, markers of muscle damage, such as creatine kinase (CK) and lactate dehydrogenase (LDH), have been shown to increase in response to a single judo match, with CK levels remaining elevated in judo athletes following a three-day training period [55]. With respect to extended duration training periods, increases in CK, fibrinogen, and markers of oxidative stress (protein carbonyls and malondialdehyde) have been shown in elite judokas undertaking twice-daily training sessions six times per week for eight weeks [44].

\subsection{Cardiovascular Function}

Only a few studies have examined blood-based markers of cardiovascular function in judo athletes, including the measurement of cardiac hormones that were found to be different from marathon runners and controls [75], establishing hemoglobin mass as being one of several discriminating factors amongst different types of youth athletes, and the non-invasive evaluation of the hemodynamic response to high-intensity exercise [93].

\section{Discussion}

\subsection{Overall Summary}

This review aimed to elaborate on the effects of judo-specific training on various health aspects among judokas and judo practitioners. Although overall health-related effects of judo training could not be completely clarified, the majority of included studies suggested a positive influence on health outcomes possibly mediated by judo training. Yet, some of the exhibited changes found in judokas and judo participants might not be beneficial. For instance, markedly increased blood pressure was noticed in judokas as reported by Casals et al. [64]. Likewise, substantial cardiac remodeling was detected in a study by Pelliccia et al. [74], and alterations on the vascular system showed a close relation of superior exercise performance on the cardiovascular system in judo. Cardiovascular adaptations depend upon the characteristics, intensity, and accumulative extent of pieces of training, with a "dose-effect relation" [94]. Observed circulatory changes illustrate significant cardiovascular adaptations induced by judo training. Whether these physiological adaptations of judokas and judo practitioners can have negative ramifications regarding cardiovascular disease development remains to be elaborated in future studies that follow judo athletes for an extended period of time. 
Data found through our review show that all judokas and judo practitioners included in this review (both male and female) showed above-average values regarding maximal oxygen consumption $\left(\mathrm{VO}_{2 \max }\right)$ for their specific age group when compared against the overall population, and not against athletes [95]. As a cornerstone of applied physiology, and the strongest predictor of cardiovascular and all-cause mortality [96], $\mathrm{VO}_{2 \max }$ is an extremely important indicator of one's health. Improvements in $\mathrm{VO}_{2 m a x}$ as a consequence of judo participation are expected due to its intermittent nature and rather demanding physiological requirements. Indeed, the interval properties of judo can be seen as a form of high-intensity interval training that can be used by a broad spectrum of the healthy population.

Unfortunately, research examining the effects of judo-specific activity and/or training on concentrations of hormones, immune and inflammatory mediators, and biomarkers of metabolic and cardiovascular function is in its infancy. Due to limited data being available regarding changes in these biomarkers in conjunction with functional health outcomes, the meaningfulness of these findings in the context of overall health is unclear.

Since judo comprises various throwing and falling techniques, it is reasonable to presume it could have an impact on bone structure. It is worth noting that judokas, along with wrestlers, demonstrated greater neck BMD values compared to endurance sports athletes such as runners, and even taekwondo athletes, which might have appeared due to the specificity of the two combat sports [20]. According to research conducted by Matsumoto et al. [83], total BMD in judokas of both genders appeared to be greater when compared to swimmers and runners. In studies where the control (non-athletes) group was included, judokas showed significantly higher values both for BMD and BMC [69]. Additionally, somewhat greater BMD values were observed in male judokas compared to female ones $[81,83]$. By summarizing the results from the retrieved studies, it is evident that judokas have enhanced bone health parameters compared to other athletes and controls in almost all measured regions.

Included studies elaborating on the body composition of judokas showed that all judokas were within the reference range of either good or acceptable body fat percentage, except for the heavyweight male athletes examined in one study [52]. Judokas also tend to have more lean mass in comparison to other athletes, and non-athletes as well [51,58]. Furthermore, each study included in our review demonstrated a range referred to as healthy values, except for the aforementioned study completed by Murata et al. [52]. Although our review included various male and female weight categories along with different competitive levels, the majority of included athletes and participants showed a healthy BMI range. In line with the above-mentioned health benefits, engaging in judo might be a suitable way to improve and maintain one's body composition, even if the practitioner is not involved professionally.

Still, judo participation is not without risks. Despite our research being focused primarily on the potential health benefits associated with judo practice, related risks of judo practice have to be mentioned. Namely, a systematic review on injuries in judo by Pocecco et al. [97] showed that during the Olympic Games in 2008 and 2012, an average injury risk of about $11-12 \%$ was detected in judo athletes. Most commonly reported injuries were sprains, strains, and contusions, usually of the knee, shoulder, and fingers, whereas being thrown was the most common way of getting injured. Acute severe injuries were quite rare and usually affected the brain and spine, whereas chronic injuries typically affected the finger joints, lower back, and ears.

In addition to injury risk, weight cycling (rapid weight loss (RWL) followed by rapid weight gain) is another factor that can contribute to health complications in professional judokas. As judo is a weight-divided sport, many athletes choose to exploit this system by manipulating their weight prior to weigh-in, so they can enter into lower weight classes, then rapidly refeed and rehydrate in the period after weigh-in and pre-match, in order to gain an advantage over lighter opponents. In a study by Artioli et al. [98] $(n=822)$, authors found that more than $90 \%$ of judokas engage in RWL prior to competitive events. 
Current literature recognizes RWL as a 5\% weight loss achieved in 5-7 days, but there are many possible variations on the same theme. This approach is prompted up to 10 times annually by judokas [98], but this is primarily contingent on the number of competitions in a given year. To achieve such RWL, judokas commonly engage in drastically reduced fluid intake, severe caloric deficit, elevated levels of intense exercise, plastic suit and heated room training, and frequent sauna use, whereas more radical methods of weight loss, such as laxative and diuretics consumption, and forced vomiting, are rare [98]. This particular method of weight reduction can lead to significant health complications, both acutely and chronically $[99,100]$. Moreover, a marked decrease in performance due to RWL-induced muscle damage has been observed [101]. Novel findings indicate that RWL can also induce acute kidney damage [102].

\subsection{Strengths and Limitations}

Perhaps one of the biggest limitations of the current study is that the majority of included studies had males as participants, and thus our findings pertain predominantly to the male population. The age set for inclusion criteria was 18-35, which narrows the results of our systematic review, and raises the question of if the benefits of judo practice outweigh the risk of potential injury in the older population. Also, included studies were highly heterogeneous in terms of study design. Throughout our study, we examined only physical aspects of judo training, and excluded important aspects of wellbeing such as diet and sleep due to unavailability of data. Further research should elaborate on the health status of recently retired judokas to see how long-term judo participation affected their health. Finally, at the beginning of the review design, we intended to conduct a more detailed meta-analysis that would have allowed us to estimate the overall effect sizes for the desired outcome. However, a meta-analysis was precluded due to the high heterogeneity of the acquired data.

This review provides comprehensive evidence for the general population to engage in what is likely a new venue for PA that goes beyond conventional PA recommendations. Regarding methodology, we applied rigorous inclusion and exclusion criteria, and meticulously analyzed each of the included articles. As a result, we examined 84 reports that had nearly 2500 participants. Our research sought to find articles published from 1996 to 2021, which enabled us to find quality articles, filter them through the Newcastle-Ottawa scale, and synthesize data acquired from nearly two and a half decades of peer-reviewed data.

\section{Conclusions}

A positive association has been found between judo participation and health benefits, including above-average $\mathrm{VO}_{2 \max }$, improved body composition, increased bone mineral density, and bone mineral content. However, since judo is a contact sport, caution should be taken, and risk/benefits analysis should be considered. Increased blood pressure, arterial adaptations, and cardiac hypertrophy in a significant number of judokas evidence that the cardiovascular system exhibits great changes. Whether these physiological adaptations of judokas and judo practitioners pose a risk for future cardiovascular diseases remains to be examined in future studies.

Future longitudinal studies with appropriate adjustment for confounding variables and self-selection are merited. These studies should aim to detect whether the positive associations between lean body mass, improved aerobic fitness, and improved bone health are due to the health benefits of judo, or the effect of self-selection and the healthy lifestyle of judokas. Moreover, dietary and recovery aspects should also be considered.

Author Contributions: Conceptualization, P.D., E.F., D.H.F. and A.B.; methodology, N.L., R.R. and A.J.W.; validation, A.M., P.D., A.P., T.T., N.L., E.F. and D.H.F.; formal analysis, N.L., R.R. and J.P.L.S.; investigation, N.L., R.R., P.D., E.F. and J.P.L.-S.; resources, A.M., A.J.W., N.L. and P.D.; data curation, N.L., A.J.W. and E.F.; writing-original N.L., R.R., E.F., J.P.L.-S., T.T., A.M. and P.D.; writingreview and editing, A.B., E.F., A.P., D.H.F. and P.D.; visualization, T.T.; supervision, A.P.; project 
administration, N.L., R.R. and T.T.; funding acquisition, P.D. All authors have read and agreed to the published version of the manuscript.

Funding: This study received support from the Serbian Ministry of Education, Science and Technological Development (179011) and the Provincial Secretariat for Higher Education and Scientific Research (142-451-2094).

Conflicts of Interest: The authors have no conflicts of interest, financial or otherwise, to disclose concerning this manuscript. The authors declare that the results of this study are presented clearly, honestly, and without fabrication, falsification, or inappropriate data manipulation.

\section{References}

1. Warburton, D.E.R.; Nicol, C.W.; Bredin, S.S.D. Health benefits of physical activity: The evidence. Can. Med. Assoc. J. 2006, 174, 801-809. [CrossRef]

2. World Health Organization. The World Health Report 2006: Working Together for Health; World Health Organization: Geneva, Switzerland, 2006.

3. Guthold, R.; Stevens, G.A.; Riley, L.M.; Bull, F.C. Worldwide trends in insufficient physical activity from 2001 to 2016 : A pooled analysis of 358 population-based surveys with 1.9 million participants. Lancet Glob. Health 2018, 6, e1077-e1086. [CrossRef]

4. Garber, C.E.; Blissmer, B.; Deschenes, M.R.; Franklin, B.A.; Lamonte, M.J.; Lee, I.M.; Nieman, D.C.; Swain, D.P. American College of Sports Medicine. American College of Sports Medicine position stand. Quantity and quality of exercise for developing and maintaining cardiorespiratory, musculoskeletal, and neuromotor fitness in apparently healthy adults: Guidance for prescribing exercise. Med. Sci. Sports Exerc. 2011, 43, 1334-1359. [CrossRef] [PubMed]

5. Lakicevic, N.; Gentile, A.; Mehrabi, S.; Cassar, S.; Parker, K.; Roklicer, R.; Bianco, A.; Drid, P. Make fitness fun: Could novelty be the key determinant for physical activity adherence? Front. Psychol. 2020, 11, 577522. [CrossRef] [PubMed]

6. Kim, S.; Greenwell, T.C.; Andrew, D.P.; Lee, J.; Mahony, D.F. An analysis of spectator motives in an individual combat sport: A study of mixed martial arts fans. Sport Mark. Q. 2008, 17, 109-119.

7. Takahashi, M. Mastering Judo: History, Philosophy, Techniques, Tactics, Training; Human Kinetics: Champaign, IL, USA, 2005; ISBN 073605099X 9780736050999.

8. Franchini, E.; Artioli, G.G.; Brito, C.J. Judo combat: Time-motion analysis and physiology. Int. J. Perform. Anal. Sport 2013, 13, 624-641. [CrossRef]

9. Franchini, E.; Brito, C.J.; Fukuda, D.H.; Artioli, G.G. The physiology of judo-specific training modalities. J. Strength Cond. Res. 2014, 28, 1474-1481. [CrossRef] [PubMed]

10. Moher, D.; Liberati, A.; Tetzlaff, J.; Altman, D.G.; Altman, D.; Antes, G.; Atkins, D.; Barbour, V.; Barrowman, N.; Berlin, J.A.; et al. Preferred reporting items for systematic reviews and meta-analyses: The PRISMA statement. PLoS Med. 2009, 6, e1000097. [CrossRef]

11. Wells, G.; Shea, B.; O'Connell, D.; Peterson, J.; Welch, V.; Losos, M.; Tugwell, P. Newcastle-Ottawa Quality Assessment Scale Cohort Studies; University of Ottawa: Ottawa, ON, Canada, 2014.

12. Ahmaidi, S.; Portero, P.; Calmet, M.; Lantz, D.; Vat, W.; Libert, J.P. Oxygen uptake and cardiorespiratory responses during selected fighting techniques in judo and kendo. Sports Med. Train. Rehabil. 1999, 9, 129-139. [CrossRef]

13. Suay, F.; Salvador, A.; González-Bono, E.; Sanchís, C.; Martínez, M.; Martínez-Sanchis, S.; Simón, V.M.; Montoro, J.B. Effects of competition and its outcome on serum testosterone, cortisol and prolactin. Psychoneuroendocrinology 1999, 24, 551-566. [CrossRef]

14. Borkowski, L.; Faff, J.; Starczewska-Czapowska, J. Evaluation of the aerobic and anaerobic fitness in judoists from the Polish National Team. Biol. Sport 2001, 18, 107-117.

15. Degoutte, F.; Jouanel, P.; Filaire, E. Energy demands during a judo match and recovery. Br. J. Sports Med. 2003, 37, 245-249. [CrossRef]

16. Salvador, A.; Suay, F.; González-Bono, E.; Serrano, M.A. Anticipatory cortisol, testosterone and psychological responses to judo competition in young men. Psychoneuroendocrinology 2003, 28, 364-375. [CrossRef]

17. Degoutte, F.; Jouanel, P.; Filaire, E. Solicitation of protein metabolism during a judo match and recovery. Sci. Sports 2004, 19, 28-33. [CrossRef]

18. Cottin, F.; Durbin, F.; Papelier, Y. Heart rate variability during cycloergometric exercise or judo wrestling eliciting the same heart rate level. Eur. J. Appl. Physiol. 2004, 91, 177-184. [CrossRef]

19. Laskowski, R.; Wysocki, K.; Multan, A.; Haga, S. Changes in cardiac structure and function among elite judoists resulting from long-term judo practice. J. Sports Med. Phys. Fit. 2008, 48, 366-370.

20. Mala, L.; Maly, T.; Zahalka, F.; Heller, J.; Hrasky, P.; Vodicka, P.; Mala, L. Differences in the morphological and physiological characteristics of senior and junior elite Czech judo athletes. Arch. Budo 2015, 11, 217-226.

21. Kujach, S.; Ziemann, E.; Grzywacz, T.; Luszczyk, M.; Smaruj, M.; Dzedzej, A.; Laskowski, R. Muscle oxygenation in response to high intensity interval exercises among high trained judokas. Isokinet. Exerc. Sci. 2016, 24, 263-275. [CrossRef]

22. Julio, U.F.; Panissa, V.L.G.; Esteves, J.V.; Cury, R.L.; Agostinho, M.F.; Franchini, E. Energy-system contributions to simulated judo matches. Int. J. Sports Physiol. Perform. 2017, 12, 676-683. [CrossRef] 
23. Paulo Lopes-Silva, J.P.; Panissa, V.L.G.; Julio, U.F.; Franchini, E. Influence of physical fitness on special judo fitness test performance. J. Strength Cond. Res. 2021, 35, 1732-1738. [CrossRef]

24. Julio, U.F.; Panissa, V.L.G.; Cury, R.L.; Agostinho, M.F.; João Esteves, V.D.C.; Franchini, E. Energy system contributions in upper and lower body wingate tests in highly trained athletes. Res. Q. Exerc. Sport 2019, 90, 244-250. [CrossRef]

25. Shiroma, S.A.; Julio, U.F.; Franchini, E. Criterion validity, reliability, and usefulness of a judo-specific maximal aerobic power test. Int. J. Sports Physiol. Perform. 2019, 14, 987-993. [CrossRef] [PubMed]

26. Sterkowicz, S.; Zuchowicz, A.; Kubica, R. Levels of anaerobic and aerobic capacity indices and results for the special fitness test in judo competitors. J. Hum. Kinet. 1999, 2, 115-135.

27. Franchini, E.; Takito, M.Y.; Nakamura, F.Y.; Matsushigue, K.A.; Peduti Dal'Molin Kiss, M.A. Effects of recovery type after a judo combat on blood lactate removal and on performance in an intermittent anaerobic task. J. Sports Med. Phys. Fit. 2003, 43, 424-431.

28. Franchini, E.; Takito, M.Y.; Kiss, M.A.P.D.M.; Sterkowicz, S. Physical fitness and anthropometrical differences between elite and non-elite judo players. Biol. Sport 2005, 22, 315-328.

29. Sbriccoli, P.; Bazzucchi, I.; Di Mario, A.; Marzattinocci, G.; Felici, F. Assessment of maximal cardiorespiratory performance and muscle power in the Italian olympic judoka. J. Strength Cond. Res. 2007, 21, 738-744. [CrossRef]

30. Franchini, E.; Bertuzzi, R.C.M.; Degaki, E.; de Campos Mello, F.; Fiebig, F.; Wagner, F.; Ferreira, L.S. Energy expenditure in different judo throwing techniques. In Proceedings of the First Joint International Pre-Olympic Conference of Sports Science and Sports Engineering; Liverpool World Academic Union: Liverpool, UK, 2008; pp. 55-60.

31. Trivic, T.; Drid, P.; Obadov, S. Aerobic capacity of male judokas in comparison with University students of the Faculty of Sport and Physical Education. Arch. Budo 2009, 5, 143-146.

32. Santos, L.; González, V.; Iscar, M.; Brime, J.I.; Fernandez-Rio, J.; Egocheaga, J.; Rodríguez, B.; Montoliu, M.Á. A new individual and specific test to determine the aerobic-anaerobic transition zone (Santos test) in competitive judokas. J. Strength Cond. Res. 2010, 24, 2419-2428. [CrossRef]

33. Santos, L.; González, V.; Iscar, M.; Brime, J.I.; Fernández-Río, J.; Rodríguez, B.; Montoliu, Á. Retesting the validity of a specific field test for judo training. J. Hum. Kinet. 2011, 29, 141-150. [CrossRef] [PubMed]

34. Franchini, E.; Sterkowicz, S.; Szmatlan-Gabrys, U.; Gabrys, T.; Garnys, M. Energy system contributions to the special judo fitness test. Int. J. Sports Physiol. Perform. 2011, 6, 334-343. [CrossRef]

35. Santos, L.; González, V.; Iscar, M.; Brime, J.I.; Fernández-Río, J.; Rodríguez, B.; Montoliu, M.Á. Physiological response of high-level female judokas measured through laboratory and field tests. retesting the validity of the santos test. J. Sports Med. Phys. Fit. 2012, 52, 237-244.

36. Radjo, I.; Trivic, T.; Bilic, A.; Atanasov, D.; Todorov, I.; Drid, P. Alteration in biomarkers of oxidative stress in judokas with different age. HealthMED 2012, 6, 3228-3232.

37. Drid, P.; Casals, C.; Mekic, A.; Radjo, I.; Stojanovic, M.; Ostojic, S.M. Fitness and anthropometric profiles of international vs. national judo medalists in half-heavyweight category. J. Strength Cond. Res. 2015, 29, 2115-2121. [CrossRef] [PubMed]

38. Schwartz, J.; Takito, M.Y.; Del Vecchio, F.B.; Antonietti, L.S.; Franchini, E. Health-related physical fitness in martial arts and combat sports practitioners. Sport Sci. Health 2015, 11, 171-180. [CrossRef]

39. Coswig, V.S.; Gentil, P.; Bueno, J.C.A.; Follmer, B.; Marques, V.A.; Del Vecchio, F.B. Physical fitness predicts technical-tactical and time-motion profile in simulated Judo and Brazilian Jiu-Jitsu matches. PeerJ 2018, 6, e4851. [CrossRef]

40. Franchini, E.; Nunes, A.V.; Moraes, J.M.; Del Vecchio, F.B. Physical fitness and anthropometrical profile of the Brazilian male judo team. J. Physiol. Anthropol. 2007, 26, 59-67. [CrossRef]

41. Drid, P.; Maksimovic, N.; Matic, R.; Obradovic, B.; Milosevic, Z.; Ostojic, S.M. Fitness profiles of elite female judokas of the Serbian national team [Profili di fitness di judoka d'elite di sesso femminile della squadra nazionale della Serbia]. Med. Sport 2009, 62, 251-263.

42. Almansba, R.; Sterkowicz, S.; Belkacem, R.; Sterkowicz-Przybycien, K.; Mahdad, D. Anthropometrical and physiological profiles of the Algerian Olympic judoists. Arch. Budo 2010, 6, 185-193.

43. Almansba, R.; Sterkowicz, S.; Sterkowicz-Przybycien, K.; Belkacem, R. Maximal oxygen uptake changes during judoist's periodization training. Arch. Budo 2010, 6, 117-122.

44. Golami Bermi, T.; Ali, P.N. The relationship between some structural properties of body and aerobic/anaerobic power in members of national female judo team. Life Sci. J. 2012, 9, 1449-1453.

45. Tartibian, B.; Nouri, H.; Azadpour, N.; Koşar, Ş.N.; Massart, A.; Filaire, E. Eight weeks judo training increases oxidative stress biomarkers and creatine kinase in male judoka. Indian J. Biochem. Biophys. 2015, 52, 281-288.

46. Garbouj, H.; Selmi, M.A.; Haj Sassi, R.; Haj Yahmed, M.; Chamari, K.; Chaouachi, A. Do maximal aerobic power and blood lactate concentration affect Specific Judo Fitness Test performance in female judo athletes? Biol. Sport 2016, 33, 367-372. [CrossRef] [PubMed]

47. Morales, J.; Franchini, E.; Garcia-Massó, X.; Solana-Tramunt, M.; Buscà, B.; González, L.M. The work endurance recovery method for quantifying training loads in Judo. Int. J. Sports Physiol. Perform. 2016, 11, 913-919. [CrossRef]

48. Arazi, H.; Noori, M.; Izadi, M. Correlation of anthropometric and bio-motor attributes with Special Judo Fitness Test in senior male judokas. IDO Mov. Cult. 2017, 17, 19-24. [CrossRef]

49. Quintero, A.M.; da Rosa Orssatto, L.B.; Pulgarín, R.D.; Follmer, B. Physical performance, body composition and somatotype in Colombian judo athletes. IDO Mov. Cult. 2019, 19, 56-63. [CrossRef] 
50. Ermiş, E.; Yilmaz, A.K.; Mayda, H.; Ermis, A. Analysis of respiratory function and muscle strength of elite judo athletes and sedentary females. J. Hum. Sport Exerc. 2019, 14, 705-710. [CrossRef]

51. Ermis, E.; Yilmaz, A.K.; Mayda, M.H. Analysis of respiratory functions and respiratory muscle strength of martial arts athletes. Int. J. Appl. Exerc. Physiol. 2019, 8, 10-17.

52. Murata, H.; Oshima, S.; Torii, S.; Taguchi, M.; Higuchi, M. Characteristics of body composition and cardiometabolic risk of Japanese male heavyweight Judo athletes. J. Physiol. Anthropol. 2016, 35, 1-6. [CrossRef]

53. Burdukiewicz, A.; Pietraszewska, J.; Andrzejewska, J.; Witkowski, K.; Stachoń, A.; Chromik, K.; Maśliński, J. Morphological differentiation and body composition in female judokas and female weightlifters in relation to the performed sport discipline. Arch. Budo 2010, 6, 111-115.

54. Yucel, O. The effects of four weeks training on leptin levels in junior female judokas. Afr. J. Microbiol. Res. 2011, 5, 2752-2754. [CrossRef]

55. Laskowski, R.; Ziemann, E.; Olek, R.; Zembron-Lacny, A. The effect of three days of judo training sessions on the inflammatory response and oxidative stress markers. J. Hum. Kinet. 2011, 30, 65-73. [CrossRef] [PubMed]

56. Smulski, W.; Wolska, B.; Jagiełło, W.; Sawczyn, S. The correlation of general and sport-specific preparation indices of elite female judo competitors with their age-somatic characteristics. Arch. Budo 2011, 7, 233-238.

57. Socha, M.; Witkowski, K.; Jonak, W.; Sobiech, K.A. Body composition and selected anthropometric traits of elite Polish female judokas in relation to the performance of right-dominant, left-dominant, or symmetrical judo techniques in vertical posture (tachi waza). Arch. Budo 2017, 12, 257-265.

58. Buśko, K.; Pastuszak, A.; Kalka, E. Body composition and somatotype of judo athletes and untrained male students as a reference group for comparison in sport. Biomed. Hum. Kinet. 2017, 9, 7-13. [CrossRef]

59. Ceylan, B.; Gurses, V.V.; Akgul, M.S.; Baydil, B.; Franchini, E. Anthropometric profile, Wingate performance and Special Judo Fitness Levels of Turkish Olympic Judo Athletes. IDO Mov. Cult. 2018, 18, 15-20. [CrossRef]

60. Mala, L.; Maly, T.; Cabell, L.; Cech, P.; Hank, M.; Coufalova, K.; Zahalka, F. Body composition and morphological limbs asymmetry in competitors in six martial arts. Int. J. Morphol. 2019, 37, 568-575. [CrossRef]

61. Franchini, E.; Huertas, J.; Sterkowicz, S.; Carratalá, V.; Gutiérrez-García, C.; Escobar-Molina, R. Anthropometrical profile of elite Spanish judoka: Comparative analysis among ages. Arch. Budo 2011, 7, 239-245.

62. Sterkowicz-Przybycień, K.; Almansba, R. Sexual dimorphism of anthropometrical measurements in judoists vs untrained subject. Sci. Sport. 2011, 26, 316-323. [CrossRef]

63. Katralli, J.; Goudar, S.S. Anthropometric profile and special judo fitness levels of Indian judo players. Asian J. Sports Med. 2012, 3 , 113-118. [CrossRef]

64. Casals, C.; Huertas, J.R.; Barranco-Ruiz, Y.; Franchini, E.; Carratalá-Deval, V.; Escobar-Molina, R. Cardiovascular risk in elite Spanish judo athletes. Arch. Budo 2017, 12, 151-157.

65. Tavares Junior, A.; Olivio Junior, J.; Goncalves, B.; Drigo, A.; Dos-Santos, J. Body composition, strength and specific physical fitness as factors to discriminate performance in judokas. Arch. Budo Sci. Martial Arts Extreme Sports 2018, 14, 117-123.

66. Shariat, A.; Shaw, B.S.; Kargarfard, M.; Shaw, I.; Lam, E.T.C. Características cineantropométricas de atletas do sexo masculino de Judô, Karatê e Taekwondo. Rev. Bras. Med. Esporte 2017, 23, 260-263. [CrossRef]

67. Kubo, J.; Chishaki, T.; Nakamura, N.; Muramatsu, T.; Yamamoto, Y.; Ito, M.; Saitou, H.; Kukidome, T. Differences in fat-free mass and muscle thicknesses at various sites according to performance level among judo athletes. J. Strength Cond. Res. 2006, 20, 654-657. [CrossRef]

68. Reale, R.; Burke, L.M.; Cox, G.R.; Slater, G. Body composition of elite olympic combat sport athletes. Eur. J. Sport Sci. 2020, 20, 147-156. [CrossRef] [PubMed]

69. Andreoli, A.; Monteleone, M.; Van Loan, M.; Promenzio, L.; Tarantino, U.; De Lorenzo, A. Effects of different sports on bone density and muscle mass in highly trained athletes. Med. Sci. Sports Exerc. 2001, 33, 507-511. [CrossRef]

70. Silva, A.M.; Fields, D.A.; Heymsfield, S.B.; Sardinha, L.B. Body composition and power changes in elite judo athletes. Int. J. Sports Med. 2010, 31, 737-741. [CrossRef]

71. Gonçalves, E.M.; Matias, C.N.; Santos, D.A.; Sardinha, L.B.; Silva, A.M. Assessment of total body water and its compartments in elite judo athletes: Comparison of bioelectrical impedance spectroscopy with dilution techniques. J. Sports Sci. 2015, 33, 634-640. [CrossRef]

72. Karagounis, P.; Maridaki, M.; Papaharalampous, X.; Prionas, G.; Baltopoulos, P. Exercise-induced arterial adaptations in elite judo athletes. J. Sport. Sci. Med. 2009, 8, 428-434.

73. Whyte, G.; George, K.; Sharma, S.; Martin, L.; Draper, N.; McKenna, W. Left ventricular structure and function in elite judo players. Clin. Exerc. Physiol. 2000, 2, 204-208.

74. Pelliccia, A.; Maron, B.J.; Culasso, F.; Spataro, A.; Caselli, G. Athlete's heart in women: Echocardiographic characterization of highly trained elite female athletes. J. Am. Med. Assoc. 1996, 276, 211-215. [CrossRef]

75. Date, H.; Imamura, T.; Onitsuka, H.; Maeno, M.; Watanabe, R.; Nishihira, K.; Matsuo, T.; Eto, T. Differential increase in natriuretic peptides in elite dynamic and static athletes. Circ. J. 2003, 67, 691-696. [CrossRef]

76. Sun, B.; Ma, J.Z.; Yong, Y.H.; Lv, Y.Y. The upper limit of physiological cardiac hypertrophy in elite male and female athletes in China. Eur. J. Appl. Physiol. 2007, 101, 457-463. [CrossRef] [PubMed] 
77. Whyte, G.P.; George, K.; Sharma, S.; Firoozi, S.; Stephens, N.; Senior, R.; McKenna, W.J. The upper limit of physiological cardiac hypertrophy in elite male and female athletes: The British experience. Eur. J. Appl. Physiol. 2004, 92, 592-597. [CrossRef] [PubMed]

78. Türkmen, M. A study on serum leptin, lipoproteins and glucose levels of judoists and cyclists of Turkey. Afr. J. Pharm. Pharmacol. 2011, 5, 532-537. [CrossRef]

79. De Oliveira, D.C.X.; Rossano Procida, I.; Das Neves Borges-Silva, C. Effect of training judo in the competition period on the plasmatic levels of leptin and pro-inflammatory cytokines in high-performance male athletes. Biol. Trace Elem. Res. 2010, 135, 345-354. [CrossRef]

80. Çelik, N.; İri, R. Effects of competition term trainings on leptin values of female judokas. Afr. J. Pharm. Pharmacol. 2011, 5, 683-687.

81. Prouteau, S.; Benhamou, L.; Courteix, D. Relationships between serum leptin and bone markers during stable weight, weight reduction and weight regain in male and female judoists. Eur. J. Endocrinol. 2006, 154, 389-395. [CrossRef]

82. Prouteau, S.; Pelle, A.; Collomp, K.; Benhamou, L.; Courteix, D. Bone density in elite judoists and effects of weight cycling on bone metabolic balance. Med. Sci. Sports Exerc. 2006, 38, 694-700. [CrossRef]

83. Matsumoto, T.; Nakagawa, S.; Nishida, S.; Hirota, R. Bone density and bone metabolic markers in active collegiate athletes: Findings in long-distance runners, judoists, and swimmers. Int. J. Sports Med. 1997, 18, 408-412. [CrossRef]

84. Bozkurt, I. Effects of exercises on bone mineral density of proximal femour region among athletes of different branches. Int. J. Phys. Sci. 2010, 5, 2705-2714.

85. Bozkurt, I. Analysis of bone mass density of lumbar spine zone of athletes. Afr. J. Biotechnol. 2010, 9, 7361-7371.

86. Agostinho, M.F.; Moreira, A.; Julio, U.F.; Marcolino, G.S.; Antunes, B.M.M.; Lira, F.S.; Franchini, E. Monitoring internal training load and salivary immune-endocrine responses during an annual judo training periodization. J. Exerc. Rehabil. 2017, 13, 68-75. [CrossRef]

87. Franchini, E.; Julio, U.F.; Panissa, V.L.G.; Lira, F.S.; Gerosa-Neto, J.; Branco, B.H.M. High-intensity intermittent training positively affects aerobic and anaerobic performance in judo athletes independently of exercise mode. Front. Physiol. 2016, 7, 268. [CrossRef]

88. Lee, N.; Kim, J.; Hyung, G.A.; Park, J.H.; Kim, S.J.; Kim, H.B.; Jung, H.S. Training effects on immune function in judoists. Asian J. Sports Med. 2015, 6. [CrossRef] [PubMed]

89. Parmigiani, S.; Bartolomucci, A.; Palanza, P.; Galli, P.; Rizzi, N.; Brain, P.F.; Volpi, R. In judo, Randori (free fight) and Kata (highly ritualized fight) differentially change plasma cortisol, testosterone, and interleukin levels in male participants. Aggress. Behav. 2006, 32, 481-489. [CrossRef]

90. Filaire, E.; Maso, F.; Sagnol, M.; Ferrand, C.; Lac, G. Anxiety, hormonal responses, and coping during a judo competition. Aggress. Behav. 2001, 27, 55-63. [CrossRef]

91. Franchini, E.; Lira, F.S.; Julio, U.F.; Antunes, B.M.; Agostinho, M.F.; Shiroma, S.A.; Gonçalves Panissa, V.L. Cytokine, physiological, technical-tactical and time structure responses in simulated judo competition. Int. J. Perform. Anal. Sport 2018, 18, 595-608. [CrossRef]

92. Chinda, D.; Umeda, T.; Shimoyama, T.; Kojima, A.; Tanabe, M.; Nakaji, S.; Sugawara, K. The acute response of neutrophil function to a bout of judo training. Luminescence 2003, 18, 278-282. [CrossRef]

93. Zhao, K.; Hohmann, A.; Chang, Y.; Zhang, B.; Pion, J.; Gao, B. Physiological, anthropometric, and motor characteristics of elite Chinese youth athletes from six different sports. Front. Physiol. 2019, 10, 405. [CrossRef]

94. MacInnis, M.J.; Gibala, M.J. Physiological adaptations to interval training and the role of exercise intensity. J. Physiol. 2017, 595, 2915-2930. [CrossRef]

95. Hoffman, J. Norms for Fitness, Performance, and Health; Human Kinetics: Champaign, IL, USA, 2006; ISBN 0736054839 9780736054836.

96. Scribbans, T.D.; Vecsey, S.; Hankinson, P.B.; Foster, W.S.; Gurd, B.J. The effect of training intensity on VO2max in young healthy adults: A meta-regression and meta-analysis. Int. J. Exerc. Sci. 2016, 9, 230-247.

97. Pocecco, E.; Ruedl, G.; Stankovic, N.; Sterkowicz, S.; Del Vecchio, F.B.; Gutiérrez-García, C.; Rousseau, R.; Wolf, M.; Kopp, M.; Miarka, B.; et al. Injuries in judo: A systematic literature review including suggestions for prevention. Br. J. Sports Med. 2013, 47, 1139-1143. [CrossRef]

98. Giannini Artioli, G.; Gualano, B.; Franchini, E.; Scagliusi, F.B.; Takesian, M.; Fuchs, M.; Lancha, A.H. Prevalence, magnitude, and methods of rapid weight loss among judo competitors. Med. Sci. Sports Exerc. 2010, 42, 436-442. [CrossRef]

99. Lakicevic, N.; Roklicer, R.; Bianco, A.; Mani, D.; Paoli, A.; Trivic, T.; Ostojic, S.M.; Milovancev, A.; Maksimovic, N.; Drid, P. Effects of rapid weight loss on judo athletes: A systematic review. Nutrients 2020, 12, 1220. [CrossRef]

100. Miles-Chan, J.; Isacco, L. Weight cycling practices in sport: A risk factor for later obesity? Obes. Rev. 2020, 22, e13188. [CrossRef] [PubMed]

101. Roklicer, R.; Lakicevic, N.; Stajer, V.; Trivic, T.; Bianco, A.; Mani, D.; Milosevic, Z.; Maksimovic, N.; Paoli, A.; Drid, P. The effects of rapid weight loss on skeletal muscle in judo athletes. J. Transl. Med. 2020, 18, 1-7. [CrossRef] [PubMed]

102. Lakicevic, N.; Paoli, A.; Roklicer, R.; Trivic, T.; Korovljev, D.; Ostojic, S.M.; Proia, P.; Bianco, A.; Drid, P. Effects of rapid weight loss on kidney function in combat sport athletes. Medicina 2021, 57, 551. [CrossRef] [PubMed] 